\title{
An efficient numerical approach for solving two-point fractional order nonlinear boundary value problems with Robin boundary conditions
}

\author{
Hyunju Kim', Junseo Lee ${ }^{2}$ and Bongsoo Jang ${ }^{2^{*}}$ (D)
}

"Correspondence:

bsjang@unist.ac.kr

${ }^{2}$ Department of Mathematical

Sciences, Ulsan National Institute of Science and Technology (UNIST)،

44919, Ulsan, Republic of Korea Full list of author information is available at the end of the article

\section{를 Springer}

\begin{abstract}
This article proposes new strategies for solving two-point Fractional order Nonlinear Boundary Value Problems (FNBVPs) with Robin Boundary Conditions (RBCs). In the new numerical schemes, a two-point FNBVP is transformed into a system of Fractional order Initial Value Problems (FIVPs) with unknown Initial Conditions (ICs). To approximate ICs in the system of FIVPs, we develop nonlinear shooting methods based on Newton's method and Halley's method using the RBC at the right end point. To deal with FIVPs in a system, we mainly employ High-order Predictor-Corrector Methods (HPCMs) with linear interpolation and quadratic interpolation (Nguyen and Jang in Fract. Calc. Appl. Anal. 20(2):447-476, 2017) into Volterra integral equations which are equivalent to FIVPs. The advantage of the proposed schemes with HPCMs is that even though they are designed for solving two-point FNBVPs, they can handle both linear and nonlinear two-point Fractional order Boundary Value Problems (FBVPs) with RBCs and have uniform convergence rates of HPCMs, $\mathcal{O}\left(h^{2}\right)$ and $\mathcal{O}\left(h^{3}\right)$ for shooting techniques with Newton's method and Halley's method, respectively. A variety of numerical examples are demonstrated to confirm the effectiveness and performance of the proposed schemes. Also we compare the accuracy and performance of our schemes with another method.
\end{abstract}

Keywords: Caputo fractional derivative; Nonlinear shooting method; Predictor-corrector scheme; Robin boundary condition

\section{Introduction}

Fractional calculus has proven to describe many phenomena in science and engineering more accurately than integer-order calculus because of the nonlocal property of the fractional derivative [2-7]. Many authors have introduced numerical methods for solving fractional differential equations arising in science and engineering. The authors in Refs. $[8,9]$ proposed a computational algorithm based on reproducing kernel Hilbert space for solving time-fractional partial differential equations in porous media and nonlinear homogeneous and nonhomogeneous time-fractional equations. In Ref. [10], a numerical method based on multiple fractional power series solution was introduced to deal with the

(c) The Author(s) 2021. This article is licensed under a Creative Commons Attribution 4.0 International License, which permits use sharing, adaptation, distribution and reproduction in any medium or format, as long as you give appropriate credit to the original author(s) and the source, provide a link to the Creative Commons licence, and indicate if changes were made. The images or other third party material in this article are included in the article's Creative Commons licence, unless indicated otherwise in a credit line to the material. If material is not included in the article's Creative Commons licence and your intended use is not permitted by statutory regulation or exceeds the permitted use, you will need to obtain permission directly from the copyright holder. To view a copy of this licence, visit http://creativecommons.org/licenses/by/4.0/. 
Schrödinger equation. The authors in Refs. [11-14] proposed several numerical methods based on collocation method, finite difference method, and L1 approximation for solving time-fractional diffusion equations. Regarding Fractional order Boundary Value Problems (FBVPs), Ref. [15] (and the references therein) investigated a Caputo fractional hybrid twopoint boundary value problem describing the thermostat models. In Ref. [16], the authors studied a fractional-order nonlocal continuum model of a Euler-Bernoulli beam whose governing equation is described as a FBVP, using the fractional finite element model.

Recently, the authors in Ref. [17] developed a spectral collocation method to deal with two-point linear multi-term FBVPs with Caputo fractional operator. In Ref. [18], the authors reformulated two-point FBVPs with a Riemann-Liouville fractional operator to a Volterra integral equation of the second kind and then developed an integral discrete scheme based on finite difference method.

However, numerical methods for solving FNBVPs with Robin Boundary Conditions (RBCs) have been paid less attention to and, in this paper, we consider the two-point Fractional order Nonlinear Boundary Value Problem (FNBVP) with RBCs:

$$
\begin{cases}D_{a}^{\alpha_{2}} y(t)=f\left(t, y, D_{a}^{\alpha_{1}} y(t)\right), & t \in[a, b] \\ a_{1} y(a)+b_{1} y^{\prime}(a)=\gamma_{1}, & a_{2} y(b)+b_{2} y^{\prime}(b)=\gamma_{2}\end{cases}
$$

where $0<\alpha_{1} \leq 1,1<\alpha_{2}<2, \alpha_{1}, \alpha_{2}, \gamma_{1}, \gamma_{2} \in \mathbb{R} . D_{a}^{\alpha_{1}}$ and $D_{a}^{\alpha_{2}}$ are Caputo fractional differentiations defined as follows.

Definition 1.1 Let $\alpha \in \mathbb{R}^{+}$. The operator $J_{a, t}^{\alpha}$, defined on $L_{1}[a, b]$ by

$$
J_{a}^{\alpha} y(t):=\frac{1}{\Gamma(\alpha)} \int_{a}^{t}(t-\tau)^{\alpha-1} y(\tau) d \tau
$$

for $a \leq t \leq b$, is called the Riemann-Liouville fractional integral operator of order $\alpha$.

We set $\alpha=0, J_{a}^{0}=I$, the identity operator.

Definition 1.2 let $\alpha \in \mathbb{R}^{+}$. The operator $D_{a}^{\alpha}$ is defined by

$$
D_{a}^{\alpha} y(t):=J_{a}^{\lceil\alpha\rceil-\alpha} D^{\lceil\alpha\rceil} y(t)=\frac{1}{\Gamma(\lceil\alpha\rceil-\alpha)} \int_{a}^{t}(t-\tau)^{\lfloor\alpha\rfloor-\alpha} y^{(\lceil\alpha\rceil)}(\tau) d \tau,
$$

where \lceil\rceil is the ceiling function and \lfloor\rfloor is the floor function.

The multi-term Caputo sense FIVP can be transformed into the system of FIVPs by Theorem 1.1 [19].

Theorem 1.1 Let us consider the following multi-term Caputo sense fractional differential equation with initial conditions:

$$
\left\{\begin{array}{l}
D_{a}^{\alpha_{n}} y(t)=f\left(t, y(t), D_{a}^{\alpha_{1}} y(t), D_{a}^{\alpha_{2}} y(t), \ldots, D_{a}^{\alpha_{n-1}} y(t)\right), \\
y^{(j)}(a)=y_{a}^{(j)}, \quad j=0,1, \ldots,\left\lfloor\alpha_{n}\right\rfloor
\end{array}\right.
$$


where $y^{(j)}(a)$ is the jth derivative at $t=a, \alpha_{n}>\alpha_{n-1}>\cdots \alpha_{1}>0, \alpha_{i}-\alpha_{i-1} \leq 1$ for all $i=$ $2,3, \ldots, n$ and $0<\alpha_{1}<1$. Then we can define $\beta_{i}$,

$$
\left\{\begin{array}{l}
\beta_{1}:=\alpha_{1}, \\
\beta_{i}:=\alpha_{i}-\alpha_{i-1}, \quad i=2,3, \ldots, n .
\end{array}\right.
$$

Then the multi-term fractional differential equation with initial conditions (2) is equivalent to the following system of fractional differential equations:

$$
\begin{aligned}
& D_{a}^{\beta_{1}} y_{1}(t)=y_{2}(t), \\
& D_{a}^{\beta_{2}} y_{2}(t)=y_{3}(t), \\
& \vdots \\
& D_{a}^{\beta_{n-1}} y_{n-1}=y_{n}(t), \\
& D_{a}^{\beta_{n}} y_{n}=f\left(t, y_{1}, y_{2}, \ldots, y_{n-2}, y_{n-1}\right),
\end{aligned}
$$

together with the initial conditions:

$$
y_{i}\left(t_{0}\right)= \begin{cases}y^{(0)} & \text { if } i=1 \\ y^{(l)} & \text { if } \alpha_{i-1}=l \in \mathbb{N} \\ 0 & \text { else, }\end{cases}
$$

in the following sense:

1. Whenever the function $y \in C^{\left\lceil\alpha_{n}\right\rceil}[a, b]$ is a solution of the multi-term equation with initial conditions (2), the vector-valued function $Y:=\left(y_{1}, \ldots, y_{n}\right)^{T}$ with

$$
y_{i}(t):= \begin{cases}y(t) & \text { if } i=1, \\ D_{a}^{\alpha_{i-1}} y(t) & \text { if } i \neq 1,\end{cases}
$$

is a solution of the system of fractional differential equations (3a) with initial conditions (3b)

2. Whenever the vector-valued function $Y:=\left(y_{1}, \ldots, y_{n}\right)^{T}$ is a solution of the system of multi-order fractional differential equations (3a) with initial conditions (3b), the function $y:=y_{1}$ is a solution of the multi-term equation with initial conditions (2).

In this paper, we propose new schemes to deal with FNBVPs and the algorithms are summarized as follows:

1 In the case that $0<\alpha_{1}<1$, we transform the FNBVP (1) with $a=0$ into a system of FIVPs using Theorem 1.1.

In the case of $\alpha_{1}=1$, i.e. the FNBVP (1) has a single term of fractional order $\alpha_{2}$, we substitute the integer order $\alpha_{1}=1$ with the fractional order $\alpha_{1}=1-\varepsilon, \varepsilon \rightarrow 0+$ so that the FNBVP satisfies the assumption, $0<\alpha_{1}<1$ in Theorem 1.1. First, the Gronwall inequality for two-term equations in [19] guarantees that the difference between the solution of FNBVP with $\alpha_{1}=1$ and with $\alpha_{1}=1-\varepsilon$ approaches 0 as $\varepsilon \rightarrow 0+$. The 
FNBVP with $\alpha_{1}=1-\varepsilon$ is transformed into a system of FIVPs and then we reduce the number of equations in the system. We prove the reduced system is equivalent to the original system as $\varepsilon \rightarrow 0+$ in Sect. 2 .

2 To deal with FIVPs, we adopt high-order predictor-corrector methods (HPCMs) with linear interpolation and quadratic interpolation [1] into Volterra integral equations which are equivalent to FIVPs.

3 ICs of the FIVPs in the system equivalent to (1) are obtained by RBC at $t=0$. But ICs include $s:=y^{\prime}(0)$ and since $s$ is unknown, we approximate $s$ by means of nonlinear shooting techniques based on Newton's method and Halley's method. The error function $\left|a_{2} y(b, s)+b_{2} y^{\prime}(b, s)-\gamma_{2}\right|$ is used to construct the root-finding problem in order to make the approximate solution to $y(t)$ satisfy the RBC at $t=b$.

4 The algorithm of the proposed shooting technique is as follows: The system of FIVPs is solved with an initial approximation to $s, s_{k}$ at the $k$ th iteration. Using the approximate solution to the system obtained by HPCMs with $s_{0}$, we find $s_{1}$ by solving Newton's (Halley's) formula. We update the approximate solution to the system with $s_{1}$ and measure the norm of the error function. We repeat this process until the norm of the error function is within the tolerance.

Similar to our proposed schemes, the authors in Refs. [20,21] introduced numerical methods for solving FBVPs with RBCs. In Refs. [20, 21], the FBVP with RBCs is turned into the FIVP by using a shooting method with a guess for the unknown IC $y(0)$ and then the FIVP is transformed into the Volterra integral equation. The integral-differential term in the Volterra integral equation is approximated by an integral discretization scheme with constant and first-order interpolating polynomials in paper [20] and [21], respectively. However, the integral discretization schemes can only handle linear FBVPs and the rate of convergence depends on the fractional order $\alpha$. This is elaborately addressed in Sect. 4 . The main advantages of our proposed schemes are as follows:

1 The proposed schemes can handle both linear and nonlinear FBVPs with general RBCs.

2 Our proposed schemes can deal with multi-term FBVPs where $0<\alpha_{1} \leq 1$ and $1<\alpha_{2}<2$.

3 Our proposed methods with HPCMs have uniform convergence rates $\mathcal{O}\left(h^{2}\right)$ and $\mathcal{O}\left(h^{3}\right)$ for shooting techniques based on Newton's method and Halley's method, respectively, with enough iterations, regardless of fractional orders thanks to the global error estimates of HPCMs in [1].

4. It is not required to solve a matrix system as Newton's method and Halley's method are applied into a system of FIVPs.

This article is organized as follows. In Sect. 2, we describe an idea about the transformation of FNBVP with RBCs (1) into a system of FIVPs. In Sect. 3, we describe nonlinear shooting methods based on Newton's method and Halley's method, to approximate unknown IC $s:=y(0)$ of FIVPs in the system. Also, we briefly mention how to apply the HPCMs into a system of FIVPs in Sect. 3. In Sect. 4, we demonstrate numerical examples verifying that the proposed shooting techniques combined with HPCMs guarantee the global convergence rates of HPCMs. We also confirm the performance and effectiveness of the proposed methods by comparing with the modified integral discretization scheme in Ref. [21]. A conclusion will be given in Sect. 5. Finally tables of numerical results and 
the linear explicit method which is an alternative method for solving FIVPs are described in the Appendix.

\section{Problem formulation}

In this section, we describe how to transform the FNBVP with RBCs (1) into a system of FIVPs according to the value of $\alpha_{1}$. Basically, we apply Theorem 1.1 with $\beta_{1}:=\alpha_{1}, \beta_{2}:=$ $1-\alpha_{1}, \beta_{3}:=\alpha_{2}-1$ to the FNBVP in the case of $0<\alpha_{1}<1$. If $\alpha_{1}$ is equal to 1 , then we replace $\alpha_{1}$ with $1-\epsilon, \epsilon \rightarrow 0+$ and set $\beta_{1}:=1-\epsilon, \beta_{2}:=\epsilon, \beta_{3}:=\alpha_{2}-1$. We reduce the size of system using the fact $\beta_{2} \rightarrow 0+$.

Case 1: $0<\alpha_{1}<1$

First, we consider a FNBVP with Dirichlet boundary conditions as follows:

$$
\left\{\begin{array}{l}
D_{0}^{\alpha_{2}} y(t)=f\left(t, y(t), D_{0}^{\alpha_{1}} y(t)\right), \quad t \in[0, b] \\
y(0)=y_{0}, \quad y(b)=y_{b}
\end{array}\right.
$$

Applying Theorem 1.1 with $\beta_{1}:=\alpha_{1}, \beta_{2}:=1-\alpha_{1}, \beta_{3}:=\alpha_{2}-1$, the FNBVP with Dirichlet boundary conditions (4) can be transformed as follows:

$$
\begin{cases}D_{0}^{\alpha_{1}} y(t)=w(t), & y(0)=y_{0}, y(b)=y_{b} \\ D_{0}^{1-\alpha_{1}} w(t)=z(t), & w(0)=0, \\ D_{0}^{\alpha_{2}-1} z(t)=f(t, y(t), w(t)), & z(0)=y^{(1)}(0) .\end{cases}
$$

From the system of fractional differential equations (5), we obtain the following system of FIVPs:

$$
\begin{cases}D_{0}^{\alpha_{1}} y(t)=w(t), & y(0)=y_{0} \\ D_{0}^{1-\alpha_{1}} w(t)=z(t), & w(0)=0, \\ D_{0}^{\alpha_{2}-1} z(t)=f(t, y(t), w(t)), & z(0)=s,\end{cases}
$$

where the IC $s$ is unknown and so needs to be approximated.

Similar to the case of Dirichlet boundary conditions, the FNBVP with RBCs (1) can be written as follows:

$$
\begin{cases}D_{0}^{\alpha_{1}} y(t)=w(t), & y(0)=y_{0}=\frac{\gamma_{1}-b_{1} s}{a_{1}} \\ D_{0}^{1-\alpha_{1}} w(t)=z(t), & w(0)=0 \\ D_{0}^{\alpha_{2}-1} z(t)=f(t, y(t), w(t)), & z(0)=s .\end{cases}
$$

Case 2: $\alpha_{1}=1$

We consider the following FBVP with Dirichlet boundary conditions:

$$
\left\{\begin{array}{l}
D_{0}^{\alpha_{2}} y(t)=f\left(t, y(t), y^{\prime}(t)\right), \quad t \in[0, b] \\
y(0)=y_{0}, \quad y(b)=y_{b},
\end{array}\right.
$$

where $1<\alpha_{2}<2, \alpha_{2} \in \mathbb{R}$. Since the fractional differential equation in (8) does not satisfy the assumption, $0<\alpha_{1}<1$ in Theorem 1.1, we cannot apply the strategy used in (4) to 
(8). So we modify the equation in (8) to meet the assumption, with the same boundary conditions as in (8) as follows:

$$
\left\{\begin{array}{l}
D_{0}^{\alpha_{2}} y(t)=f\left(t, y(t), D_{0}^{1-\varepsilon} y(t)\right), \quad t \in[0, b] \\
y(0)=y_{0}, \quad y(b)=y_{b}
\end{array}\right.
$$

where $\alpha_{2} \in(1,2), \epsilon \rightarrow 0+$. By Lemma 2.1, solutions of the two FBVPs (8) and (9) are approximately equal and the absolute error depends on $\epsilon$.

Lemma 2.1 (First Gronwall inequality for two-term equations in [19]) Let $\alpha_{2}>0$ and $\alpha_{1}, \tilde{\alpha}_{1} \in\left(0, \alpha_{2}\right)$ be chosen so that the equation

$$
D_{0}^{\alpha_{2}} y(t)=f\left(t, y(t), D_{0}^{\alpha_{1}} y(t)\right)
$$

subject to the initial conditions

$$
y(0)=y_{0}, \quad y^{(1)}(0)=y_{0}^{1}, \quad \ldots, \quad y^{\left(\left\lceil\alpha_{2}\right\rceil-1\right)}(0)=y_{0}^{\left\lceil\alpha_{2}\right\rceil-1}
$$

and

$$
D_{0}^{\alpha_{2}} z(t)=f\left(t, z(t), D_{0}^{\tilde{\alpha}_{1}} \tilde{z}(t)\right)
$$

subject to the same initial conditions

$$
z(0)=y_{0}, \quad z^{(1)}(0)=y_{0}^{1}, \quad \ldots, \quad z^{\left(\left\lceil\alpha_{2}\right\rceil-1\right)}(0)=y_{0}^{\left\lceil\alpha_{2}\right\rceil-1}
$$

(where $f$ satisfies a Lipschitz condition in its second and third arguments on a suitable domain) has unique continuous solutions $y, z:[0, T] \rightarrow \mathbb{R}$. We assume further that $\left\lfloor\alpha_{1}\right\rfloor=$ $\left\lfloor\tilde{\alpha_{1}}\right\rfloor$. Then there exist constants $K_{1}$ and $K_{2}$ such that

$$
|y(t)-z(t)| \leq K_{1}\left|\alpha_{1}-\tilde{\alpha}_{1}\right| E_{\alpha_{n}}\left(K_{2} T^{\alpha_{2}}\right), \quad \forall t \in[0, T],
$$

where $E_{\alpha_{n}}$ denotes the Mittag-Leffler function of order $\alpha_{n}$.

Applying Theorem 1.1 to (9), we obtain the following system of FIVPs:

$$
\begin{cases}D_{0}^{1-\epsilon} y(t)=w(t), & y(0)=y_{0}, \\ D_{0}^{\epsilon} w(t)=z(t), & w(0)=0, \\ D_{0}^{\alpha_{2}-1} z(t)=f(t, y(t), w(t)), & z(0)=s .\end{cases}
$$

Now, we show that the system of FIVPs (10) is equivalent to the following system as $\epsilon \rightarrow 0$ using Lemmas 2.2 through 2.4 and Theorem 2.1:

$$
\begin{cases}D_{0}^{1-\epsilon} \tilde{y}(t)=\tilde{z}(t), & \tilde{y}(0)=y_{0} \\ D_{0}^{\alpha_{2}-1} \tilde{z}(t)=f(t, \tilde{y}(t), \tilde{z}(t)), & \tilde{z}(0)=s .\end{cases}
$$


Lemma 2.2 (Theorem 2.10 in [19]) Let $f \in C[a, b]$ and $\alpha \geq 0$. Moreover, assume that $\alpha_{k}$ is a sequence of positive numbers such that $\alpha_{k} \rightarrow \alpha$ as $k \rightarrow \infty$. Then, for every $\delta>0$,

$$
\lim _{k \rightarrow \infty} \sup _{t \in[a+\delta, b]}\left|J_{a}^{\alpha_{k}} f(t)-J_{a}^{\alpha} f(t)\right|=0
$$

Lemma 2.3 (Lemma 6.19 in [19]) Let $\alpha, T, \phi_{1}, \phi_{2} \in \mathbb{R}^{+}$. Moreover, assume that $\delta:[0, T] \rightarrow$ $\mathbb{R}$ is a continuous function satisfying the inequality

$$
|\delta(t)| \leq \phi_{1}+\frac{\phi_{2}}{\Gamma(\alpha)} \int_{0}^{t}(t-\tau)^{\alpha-1}|\delta(\tau)| d \tau, \quad \forall t \in[0, T]
$$

Then

$$
|\delta(t)| \leq \phi_{1} E_{\alpha}\left(\phi_{2} t^{\alpha}\right), \quad \forall t \in[0, T]
$$

Lemma 2.4 Let $0<\gamma \leq \alpha \leq \beta$. Then, for any $t \in[a, b]$,

$$
\left|J_{a}^{\alpha} y(t)-J_{a}^{\beta} y(t)\right| \leq\left[\frac{\Gamma(\gamma)}{\Gamma(\alpha)}(b-a)^{\alpha-\gamma}+\frac{\Gamma(\gamma)}{\Gamma(\beta)}(b-a)^{\beta-\gamma}\right] J_{a}^{\gamma}|y(t)| .
$$

Proof By Definition 1.1,

$$
\begin{aligned}
J_{a}^{\alpha} y(t)+J_{a}^{\beta} y(t)= & \frac{1}{\Gamma(\alpha)} \int_{a}^{t}(t-\tau)^{\alpha-1} y(\tau) d \tau+\frac{1}{\Gamma(\beta)} \int_{a}^{t}(t-\tau)^{\beta-1} y(\tau) d \tau \\
= & \frac{1}{\Gamma(\alpha)} \int_{a}^{t}(t-\tau)^{\gamma-1+(\alpha-\gamma)} y(\tau) d \tau \\
& +\frac{1}{\Gamma(\beta)} \int_{a}^{t}(t-\tau)^{\gamma-1+(\beta-\gamma)} y(\tau) d s .
\end{aligned}
$$

Then

$$
\begin{aligned}
\left|J_{a}^{\alpha} y(t)-J_{a}^{\beta} y(t)\right| \leq & \frac{(b-a)^{\alpha-\gamma}}{\Gamma(\alpha)} \int_{a}^{t}(t-\tau)^{\gamma-1}|y(\tau)| d \tau \\
& +\frac{(b-a)^{\beta-\gamma}}{\Gamma(\beta)} \int_{a}^{t}(t-\tau)^{\gamma-1}|y(\tau)| d \tau \\
\leq & {\left[\frac{\Gamma(\gamma)}{\Gamma(\alpha)}(b-a)^{\alpha-\gamma}+\frac{\Gamma(\gamma)}{\Gamma(\beta)}(b-a)^{\beta-\gamma}\right] \frac{1}{\Gamma(\gamma)} \int_{a}^{t}(t-\tau)^{\gamma-1}|y(\tau)| d \tau } \\
\leq & {\left[\frac{\Gamma(\gamma)}{\Gamma(\alpha)}(b-a)^{\alpha-\gamma}+\frac{\Gamma(\gamma)}{\Gamma(\beta)}(b-a)^{\beta-\gamma}\right] J_{a}^{\gamma}|y(t)| . }
\end{aligned}
$$

Theorem 2.1 Let $1<\alpha_{2}<2,{ }^{\forall} T \in \mathbb{R}^{+}$and $f:[0, T] \times \mathbb{R} \times \mathbb{R} \rightarrow \mathbb{R}$ satisfies the Lipschiz condition in its second and third arguments on a suitable domain. Then we have the following inequality:

$$
\left|f\left(t, x_{1}, y_{1}\right)-f\left(t, x_{2}, y_{2}\right)\right| \leq L\left(\left|x_{2}-x_{1}\right|+\left|y_{2}-y_{1}\right|\right)
$$

where ${ }^{\forall} t \in[0, T], x_{1}, x_{2}, y_{1}, y_{2}:[0, T] \rightarrow \mathbb{R}$ and $0<L$. 
If, for any $0<\epsilon \ll 1, \hat{y}$ and $\tilde{y}$ are solutions of the following systems, respectively:

$$
\begin{cases}D_{0}^{1-\epsilon} \hat{y}(t)=w(t), & y(0)=y_{0}, \\ D_{0}^{\epsilon} w(t)=z(t), & w(0)=0, \\ D_{0}^{\alpha_{2}-1} z(t)=f(t, \hat{y}(t), w(t)), & z(0)=s,\end{cases}
$$

and

$$
\begin{cases}D_{0}^{1-\epsilon} \tilde{y}(t)=\tilde{z}(t), & \tilde{y}(0)=y_{0}, \\ D_{0}^{\alpha_{2}-1} \tilde{z}(t)=f(t, \tilde{y}(t), \tilde{z}(t)), & \tilde{z}(0)=s\end{cases}
$$

then

$$
|\hat{y}(t)-\tilde{y}(t)| \rightarrow 0, \quad \text { as } \epsilon \rightarrow 0 .
$$

Proof By Lemma 6.2 in [19], FIVPs $D_{0}^{1-\epsilon} \hat{y}(t)=w(t), y(0)=y_{0}$, and $D_{0}^{1-\epsilon} \tilde{y}(t)=\tilde{z}(t), \tilde{y}(0)=y_{0}$ are equivalent to Volterra integral equations of the second kind, respectively, as follows:

$$
\hat{y}(t)=y_{0}+J_{0}^{1-\epsilon} w(t), \quad \tilde{y}(t)=y_{0}+J_{0}^{1-\epsilon} \tilde{z}(t) .
$$

Then $\hat{y}(t)-\tilde{y}(t)$ can be expressed as a Riemann-Liouville fractional integral of $w(t)-\tilde{z}(t)$ as follows:

$$
\hat{y}(t)-\tilde{y}(t)=J_{0}^{1-\epsilon}(w(t)-\tilde{z}(t))
$$

Since $w(t)=J^{\epsilon} z(t)$ by Lemma 6.2 in [19] and rewriting $w(t)-\tilde{z}(t)$ as $w(t)-J_{0}^{\epsilon} \tilde{z}(t)+J_{0}^{\epsilon} \tilde{z}(t)-$ $\tilde{z}(t)$, we obtain the following inequality:

$$
|w(t)-\tilde{z}(t)| \leq J_{0}^{\epsilon}|z(t)-\tilde{z}(t)|+\left|J_{0}^{\epsilon} \tilde{z}(t)-\tilde{z}(t)\right| .
$$

Since $D_{0}^{\alpha_{2}-1} z(t)=f(t, \hat{y}(t), w(t))$ and $D_{0}^{\alpha_{2}-1} \tilde{z}(t)=f(t, \tilde{y}(t), \tilde{z}(t))$ are equivalent to Volterra integral equations $z(t)=s+J_{0}^{\alpha_{2}-1} f(t, \hat{y}(t), w(t))$ and $\tilde{z}(t)=s+J_{0}^{\alpha_{2}-1} f(t, \tilde{y}(t), w(t))$ by Lemma 6.2 in [19] respectively, using the Lipschitz condition, (12), and (13), we obtain the following inequalities:

$$
\begin{aligned}
|z(t)-\tilde{z}(t)|= & \left|J_{0}^{\alpha_{2}-1}[f(t, \hat{y}(t), w(t))-f(t, \tilde{y}(t), \tilde{z}(t))]\right| \\
\leq & J_{0}^{\alpha_{2}-1}|f(t, \hat{y}(t), w(t))-f(t, \tilde{y}(t), \tilde{z}(t))| \\
\leq & L J_{0}^{\alpha_{2}-1}(|\hat{y}(t)-\tilde{y}(t)|+|w(t)-\tilde{z}(t)|) \\
\leq & L J_{0}^{\alpha_{2}-1}\left[J_{0}^{1-\epsilon}|w(t)-\tilde{z}(t)|+J_{0}^{\epsilon}|z(t)-\tilde{z}(t)|\right. \\
& \left.+\left|J_{0}^{\epsilon} \tilde{z}(t)-\tilde{z}(t)\right|\right] .
\end{aligned}
$$

Since $1<\alpha_{2}<2$ and $\Gamma\left(\alpha_{2}\right)=\left(\alpha_{2}-1\right) \Gamma\left(\alpha_{2}-1\right)$, we have the following inequalities for $J_{0}^{\alpha_{2}-1}\left|J^{\epsilon} \tilde{z}(t)-\tilde{z}(t)\right|:$

$$
J_{0}^{\alpha_{2}-1}\left|J^{\epsilon} \tilde{z}(t)-\tilde{z}(t)\right|=\frac{1}{\Gamma\left(\alpha_{2}-1\right)} \int_{0}^{t}(t-\tau)^{\alpha_{2}-2}\left|J_{0}^{\epsilon} \tilde{z}(t)-\tilde{z}(t)\right| d \tau
$$




$$
\begin{aligned}
& \leq \frac{T^{\alpha_{2}-1}}{\Gamma\left(\alpha_{2}-1\right)}\left\|J_{0}^{\epsilon} \tilde{z}(t)-\tilde{z}(t)\right\|_{\infty} \\
& \leq \frac{T^{\alpha_{2}-1}}{\Gamma\left(\alpha_{2}\right)}\left\|J_{0}^{\epsilon} \tilde{z}(t)-\tilde{z}(t)\right\|_{\infty} .
\end{aligned}
$$

Similarly, we can obtain the following inequality:

$$
J_{0}^{\alpha_{2}-\epsilon}\left|J_{0}^{\epsilon} \tilde{z}(t)-\tilde{z}(t)\right| \leq \frac{T^{\alpha_{2}-\epsilon}}{\Gamma\left(\alpha_{2}-\epsilon+1\right)}\left\|J_{0}^{\epsilon} \tilde{z}(t)-\tilde{z}(t)\right\|_{\infty} .
$$

Applying the inequality (16) into (14), we have the following inequality:

$$
\begin{aligned}
|z(t)-\tilde{z}(t)| \leq & L\left[J_{0}^{\alpha_{2}-\epsilon}|w(t)-\tilde{z}(t)|+J_{0}^{\alpha_{2}-1+\epsilon}|z(t)-\tilde{z}(t)|\right. \\
& \left.+\frac{T^{\alpha_{2}-1}}{\Gamma\left(\alpha_{2}\right)}\left\|J_{0}^{\epsilon} \tilde{z}(t)-\tilde{z}(t)\right\|_{\infty}\right] .
\end{aligned}
$$

Using the inequalities (13) and (17), we have the following inequalities:

$$
\begin{aligned}
J_{0}^{\alpha_{2}-\epsilon}|w(t)-\tilde{z}(t)| \leq & J_{0}^{\alpha_{2}-\epsilon}\left(J_{0}^{\epsilon}|z(t)-\tilde{z}(t)|+\left|J_{0}^{\epsilon} \tilde{z}(t)-\tilde{z}(t)\right|\right) \\
\leq & J_{0}^{\alpha_{2}}|z(t)-\tilde{z}(t)| \\
& +\frac{T^{\alpha_{2}-\epsilon}}{\Gamma\left(\alpha_{2}-\epsilon+1\right)}\left\|J_{0}^{\epsilon} \tilde{z}(t)-\tilde{z}(t)\right\|_{\infty}
\end{aligned}
$$

Applying the inequality (19) into (18) and using Lemma 2.4 with $\gamma=\alpha_{2}-1, \alpha=\alpha_{2}-1+\epsilon$, $\beta=\alpha_{2}$, we have the following inequalities:

$$
\begin{aligned}
|z(t)-\tilde{z}(t)| \leq & L\left[J_{0}^{\alpha_{2}}|z(t)-\tilde{z}(t)|+\frac{T^{\alpha_{2}-\epsilon}}{\Gamma\left(\alpha_{2}-\epsilon+1\right)}\left\|J_{0}^{\epsilon} \tilde{z}(t)-\tilde{z}(t)\right\|_{\infty}\right. \\
& \left.+J_{0}^{\alpha_{2}-1+\epsilon}|z(t)-\tilde{z}(t)|+\frac{T^{\alpha_{2}-1}}{\Gamma\left(\alpha_{2}\right)}\left\|J_{0}^{\epsilon} \tilde{z}(t)-\tilde{z}(t)\right\|_{\infty}\right] \\
= & L\left[J_{0}^{\alpha_{2}-\epsilon}|z(t)-\tilde{z}(t)|+J_{0}^{\alpha_{2}-1+\epsilon}|z(t)-\tilde{z}(t)|\right. \\
& \left.+\left\{\frac{T^{\alpha_{2}-\epsilon}}{\Gamma\left(\alpha_{2}-\epsilon+1\right)}+\frac{T^{\alpha_{2}-1}}{\Gamma\left(\alpha_{2}\right)}\right\}\left\|J_{0}^{\epsilon} \tilde{z}(t)-\tilde{z}(t)\right\|_{\infty}\right] \\
\leq & L\left[\left\{\frac{\Gamma\left(\alpha_{2}-1\right)}{\Gamma\left(\alpha_{2}-1+\epsilon\right)} T^{\epsilon}+\frac{\Gamma\left(\alpha_{2}-1\right)}{\Gamma\left(\alpha_{2}\right)} T\right\} J_{0}^{\alpha_{2}-1}|z(t)-\tilde{z}(t)|\right. \\
& \left.+\left\{\frac{T^{\alpha_{2}-\epsilon}}{\Gamma\left(\alpha_{2}-\epsilon+1\right)}+\frac{T^{\alpha_{2}-1}}{\Gamma\left(\alpha_{2}\right)}\right\}\left\|J_{0}^{\epsilon} \tilde{z}(t)-\tilde{z}(t)\right\|_{\infty}\right],
\end{aligned}
$$

so that

$$
|z(t)-\tilde{z}(t)| \leq L C_{\epsilon}^{1} J_{0}^{\alpha_{2}-1}|z(t)-\tilde{z}(t)|+L C_{\epsilon}^{2}\left\|J_{0}^{\epsilon} \tilde{z}(t)-\tilde{z}(t)\right\|_{\infty}
$$

where

$$
C_{\epsilon}^{1} \equiv \frac{\Gamma\left(\alpha_{2}-1\right)}{\Gamma\left(\alpha_{2}-1+\epsilon\right)} T^{\epsilon}+\frac{\Gamma\left(\alpha_{2}-1\right)}{\Gamma\left(\alpha_{2}\right)} T, \quad C_{\epsilon}^{2} \equiv \frac{T^{\alpha_{2}-\epsilon}}{\Gamma\left(\alpha_{2}-\epsilon+1\right)}+\frac{T^{\alpha_{2}-1}}{\Gamma\left(\alpha_{2}\right)} .
$$


Applying Lemma 2.3 to inequality (20), we obtain the following inequality:

$$
|z(t)-\tilde{z}(t)| \leq L C_{\epsilon}^{2}\left\|J_{0}^{\epsilon} \tilde{z}(t)-\tilde{z}(t)\right\|_{\infty} E_{\alpha_{2}-1}\left[L C_{\epsilon}^{1} T^{\alpha_{2}-1}\right] .
$$

Therefore,

$$
\begin{aligned}
|\tilde{y}(t)-\hat{y}(t)| \leq & J_{0}^{1-\epsilon}|w(t)-\tilde{z}(t)| \\
\leq & J_{0}^{1}|z(t)-\tilde{z}(t)|+J_{0}^{1-\epsilon}\left|J_{0}^{\epsilon} \tilde{z}(t)-\tilde{z}(t)\right| \\
\leq & T|z(t)-\tilde{z}(t)|+\frac{T^{1-\epsilon}}{\Gamma(2-\epsilon)}\left\|J_{0}^{\epsilon} \tilde{z}(t)-\tilde{z}(t)\right\|_{\infty} \\
\leq & T L C_{\epsilon}^{2}\left\|J_{0}^{\epsilon} \tilde{z}(t)-\tilde{z}(t)\right\|_{\infty} E_{\alpha_{2}-1}\left[L C_{\epsilon}^{1} T^{\alpha_{2}-1}\right] \\
& +\frac{T^{1-\epsilon}}{\Gamma(2-\epsilon)}\left\|J_{0}^{\epsilon} \tilde{z}(t)-\tilde{z}(t)\right\|_{\infty} \\
= & {\left[T L C_{\epsilon}^{2} E_{\alpha_{2}-1}\left[L C_{\epsilon}^{1} T^{\alpha_{2}-1}\right]+\frac{T^{1-\epsilon}}{\Gamma(2-\epsilon)}\right]\left\|J_{0}^{\epsilon} \tilde{z}(t)-\tilde{z}(t)\right\|_{\infty} . }
\end{aligned}
$$

We have $C_{\epsilon} \equiv T L C_{\epsilon}^{2} E_{\alpha_{2}-1}\left[L C_{\epsilon}^{1} T^{\alpha_{2}-1}\right]+\frac{T^{1-\epsilon}}{\Gamma(2-\epsilon)}$. Then

$$
|\tilde{y}(t)-\hat{y}(t)| \leq C_{\epsilon}\left\|J_{0}^{\epsilon} \tilde{z}(t)-\tilde{z}(t)\right\|_{\infty}
$$

Thus, by Lemma 2.2,

$$
|\tilde{y}(t)-\hat{y}(t)| \leq C_{\epsilon}\left\|J_{0}^{\epsilon} \tilde{z}(t)-\tilde{z}(t)\right\|_{\infty} \rightarrow 0, \quad \text { as } \epsilon \rightarrow 0 .
$$

For the FNBVP with RBCs (1), similarly, by Lemma 2.1 and using Theorem 1.1, we obtain the following FIVPs:

$$
\begin{cases}D_{0}^{1-\epsilon} y(t)=w(t), & y(0)=\frac{\gamma_{1}-b_{1} s}{a_{1}}, \\ D_{0}^{\epsilon} w(t)=z(t), & w(0)=0, \\ D_{0}^{\alpha_{2}-1} z(t)=f(t, y(t), w(t)), & z(0)=s .\end{cases}
$$

The system (21) can be reduced as follows:

$$
\begin{cases}D_{0}^{1-\epsilon} \tilde{y}(t)=\tilde{z}(t), & \tilde{y}(0)=\frac{\gamma_{1}-b_{1} s}{a_{1}}, \\ D_{0}^{\alpha_{2}-1} \tilde{z}(t)=f(t, \tilde{y}(t), \tilde{z}(t)), & \tilde{z}(0)=s .\end{cases}
$$

\section{Nonlinear shooting methods and high-order predictor-corrector methods}

FBVPs have been transformed to systems of FIVPs in Sect. 2. Before we address how to deal with systems of FIVPs (6), (7), (11), and (22) using High-order Predictor-Corrector Methods (HPCMs), the unknown IC $z(0)=s$ should be handled first. In this section, we describe two nonlinear shooting techniques based on Newton's method and Halley's method to approximate $s$. Both Newton's formula and Halley's formula are designed to determine the solution of a system of FIVPs satisfying the RBC at the right end point of an interval. Without loss of generality, we consider the system of FIVPs (7) that is equivalent to the FNBVP with RBCs (1). 
In order that the RBC at the right end point $a_{2} y(b)+b_{2} y^{\prime}(b)=\gamma_{2}$ is involved in approximating $s$, we define $y(s):=\left.y(s, t)\right|_{t=b}$ and let the error function be $F(s):=a_{2} y(s)+b_{2} \frac{\partial}{\partial t} y(s)-$ $\gamma_{2}$. We approximate the solution of the root-finding problem $F(s)=0$ by using Newton's method and Halley's method, respectively. For convenience, we denote

$$
g_{s}(t)=\frac{\partial g(s, t)}{\partial s}, \quad g_{s s}=\frac{\partial^{2} g(s, t)}{\partial s^{2}}
$$

throughout this section.

\subsection{Shooting with Newton's method}

The conventional Newton formula for $F(s)=0$ can be expressed as follows:

$$
s_{k+1}=s_{k}-\frac{F\left(s_{k}\right)}{F_{s}\left(s_{k}\right)}, \quad k=0,1,2, \ldots, m
$$

where $m$ is the maximum number of iterations and

$$
\begin{aligned}
F_{s}\left(s_{k}\right) & =\left.\frac{\partial F}{\partial s}(s)\right|_{s=s_{k}, t=b} \\
& =\left.a_{2} \frac{\partial y}{\partial s}(s, t)\right|_{s=s_{k}, t=b}+\left.b_{2} \frac{\partial}{\partial s}\left[\frac{\partial y}{\partial t}(s, t)\right]\right|_{s=s_{k}, t=b} .
\end{aligned}
$$

Observing $y_{s}\left(s_{k}\right)$ and $y_{t s}\left(s_{k}\right)$, it turns out that they are equal to $\left.\frac{\partial}{\partial s} y(t)\right|_{s=s_{k}, t=b}$ and $\left.\frac{\partial}{\partial s} z(t)\right|_{s=s_{k}, t=b}$, respectively, in the system of FIVPs (7). Thus we solve the following system obtained from the system of FIVPs (7) by applying the operator $\frac{\partial}{\partial s}$ using HPCMs for each $k$ :

$$
\begin{cases}D_{0}^{\alpha_{1}} y_{s}(t)=w_{s}(t), & y_{s}(0)=-b_{1} / a_{1} \\ D_{0}^{1-\alpha_{1}} w_{s}(t)=z_{s}(t), & w_{s}(0)=0 \\ D_{0}^{\alpha_{2}-1} z_{s}(t)=f_{s}(t, y(t), w(t)), & z_{s}(0)=1\end{cases}
$$

Since both $t$ and $s$ are independent variables, $f_{s}(t, y(t), w(t))$ can be written as

$$
f_{s}(t, y(t), w(t))=f_{y} \cdot y_{s}(t)+f_{w} \cdot w_{s}(t)
$$

The detailed description of HPCMs dealing with a system of FIVPs is in Sect. 3.3. By solving the system (25), $s_{k+1}$ in Newton's formula (23) is computed. Using the updated approximate value of IC $s, s_{k+1}$, we update approximate solutions of systems of FIVPs (6), (7), (11), and (22). Repeating this process, we obtain an $s_{k}$ having an acceptable error of the root-finding problem $F(s)=0$ at an appropriate number of iterations $k$.

\subsection{Shooting with Halley's method}

The conventional Halley formula for $F(s)=0$ is as follows:

$$
s_{k+1}=s_{k}-\frac{2 F\left(s_{k}\right) F_{s}\left(s_{k}\right)}{2 F_{s}^{2}\left(s_{k}\right)-F\left(s_{k}\right) F_{s s}\left(s_{k}\right)}, \quad k=0,1,2, \ldots, m,
$$


where $F_{s}\left(s_{k}\right)$ is described in (24) and

$$
\begin{aligned}
F_{s s}\left(s_{k}\right) & =\left.\frac{\partial^{2} F}{\partial s^{2}}(s)\right|_{s=s_{k}, t=b} \\
& =\left.a_{2} \frac{\partial^{2} y}{\partial s^{2}}(s, t)\right|_{s=s_{k}, t=b}+\left.b_{2} \frac{\partial^{2}}{\partial s^{2}}\left[\frac{\partial y}{\partial t}(s, t)\right]\right|_{s=s_{k}, t=b} .
\end{aligned}
$$

Similar to the way we found $y_{s}\left(s_{k}\right)$ and $y_{t s}\left(s_{k}\right)$ in the shooting with Newton's method, we find $y_{s s}\left(s_{k}\right)$ and $z_{s s}\left(s_{k}\right)$ by solving the following system of FIVPs obtained by applying the operator $\frac{\partial^{2}}{\partial s^{2}}$ using HPCMs for each $k$ :

$$
\begin{cases}D_{a}^{\alpha_{1}} y_{s s}(t)=w_{s s}(t), & y_{s s}(0)=0, \\ D_{a}^{1-\alpha_{1}} w_{s s}(t)=z_{s s}(t), & w_{s s}(0)=0, \\ D_{a}^{\alpha_{2}-1} z_{s s}(t)=f_{s s}(t, y(t), w(t)), & z_{s s}(0)=0 .\end{cases}
$$

Since $t$ and $s$ are independent variables, $f_{s s}(t, y(t), w(t))$ can be written as

$$
f_{y} \cdot y_{s s}(t)+f_{w} \cdot w_{s s}(t)+f_{y y} \cdot y_{s}(t)^{2}+f_{w w} \cdot w_{s}(t)^{2}+f_{w y} \cdot w_{s}(t) y_{s}(t)
$$

\subsection{High-order predictor-corrector methods for system of FIVPs}

In order to find a $s_{k}$ with an acceptable accuracy, we iteratively solve systems of FIVPs (25) or (28). Once we find the $s_{k}$, we solve systems of FIVPs (6), (7), (11), or (22). In this subsection, we describe how to deal with those systems of FIVPs using High-order PredictorCorrector Methods (HPCMs) introduced in Ref. [1]. Without loss of generality, we consider the following FIVP:

$$
\left\{\begin{array}{l}
D_{0}^{\alpha} y(t)=f(t, y(t)), \quad t \in[0, b] \\
D^{(i)} y(0)=c_{i}, \quad i=0, \ldots,\lfloor\alpha\rfloor
\end{array}\right.
$$

For convenience, let us denote $y_{j}$ as approximated value of $y\left(t_{j}\right)$ except for $y_{0}=c_{0}$ and let $f_{j} \equiv f\left(t_{j}, y_{j}\right), y_{j}^{c}$ be a corrector of $y_{j}, y_{j}^{p}$ be a predictor of $y_{j}$, and $f_{j}^{p} \equiv f\left(t_{j}, y_{j}^{p}\right), j=1, \ldots, N$. If $j=0$ then $f_{0}=f\left(0, c_{0}\right)$. We divide the domain $\Omega$ as follows:

$$
\Phi_{N}:=\left\{t_{j} \mid a=t_{0}<\cdots<t_{j}<\cdots<t_{n}<t_{n+1}<\cdots<t_{N}=b\right\} .
$$

For simplicity, let the step size be uniform, which means $t_{j+1}-t_{j}=h, j=0,1, \ldots, N-1$. Then (30) can be rewritten at time $t_{n+1}$ as follows:

$$
y\left(t_{n+1}\right)=g\left(t_{n+1}\right)+\frac{1}{\Gamma(\alpha)} \sum_{j=0}^{n} \int_{t_{j}}^{t_{j+1}}\left(t_{n+1}-\tau\right)^{\alpha-1} f(\tau, y(\tau)) d \tau,
$$

where $g\left(t_{n+1}\right)=\sum_{i=0}^{\lfloor\alpha\rfloor} \frac{\left(t_{n+1}\right)^{i}}{i !} c_{i}$. We interpolate $f(\tau, y(\tau))$ using linear or quadratic Lagrange polynomials over each interval $I_{j}=\left[t_{j}, t_{j+1}\right], j=0,1, \ldots, N-1$. Then we obtain the following predictor-corrector schemes. 
1 HPCM with linear Lagrange polynomial:

$$
\begin{aligned}
y_{n+1}^{c}= & g\left(t_{n+1}\right)+\frac{1}{\Gamma(\alpha)}\left[\sum_{j=0}^{n-1}\left(B_{n+1}^{1, j} f_{j}+B_{n+1}^{2, j} f_{j+1}\right)\right. \\
& \left.+B_{n+1}^{1, n} f_{n}+B_{n+1}^{2, n} f_{n+1}^{P}\right],
\end{aligned}
$$

where

$$
\begin{aligned}
& B_{n+1}^{1, j}=\frac{1}{h} \int_{t_{j}}^{t_{j+1}}\left(t_{n+1}-\tau\right)^{\alpha-1}\left(t_{j+1}-\tau\right) d \tau, \\
& B_{n+1}^{2, j}=-\frac{1}{h} \int_{t_{j}}^{t_{j+1}}\left(t_{n+1}-\tau\right)^{\alpha-1}\left(t_{j}-\tau\right) d \tau, \\
& y_{n+1}^{P}=g\left(t_{n+1}\right)+G_{\alpha, f}\left(t_{n+1}\right)+b_{n+1}^{1} f_{n-1}+b_{n+1}^{2} f_{n}, \\
& b_{n+1}^{1}=\frac{1}{h} \int_{t_{n}}^{t_{n+1}}\left(t_{n+1}-\tau\right)^{\alpha-1}\left(t_{n}-\tau\right) d \tau, \\
& b_{n+1}^{2}=-\frac{1}{h} \int_{t_{n}}^{t_{n+1}}\left(t_{n+1}-\tau\right)^{\alpha-1}\left(t_{n-1}-\tau\right) d \tau, \\
& G_{\alpha, f}\left(t_{n+1}\right)=\frac{1}{\Gamma(\alpha)} \sum_{j=0}^{n-1}\left(B_{n+1}^{1, j} f_{j}+B_{n+1}^{2, j} f_{j+1}\right) .
\end{aligned}
$$

2 HPCM with quadratic Lagrange polynomial:

$$
\begin{aligned}
y_{n+1}^{c}= & g\left(t_{n+1}\right)+\frac{1}{\Gamma(\alpha)}\left[A_{n+1}^{1,0} f_{0}+A_{n+1}^{2,0} f_{1 / 2}+A_{n+1}^{3,0} f_{1}\right. \\
& +\sum_{j=1}^{n-1}\left(A_{n+1}^{1, j} f_{j-1}+A_{n+1}^{2, j} f_{j}+A_{n+1}^{3, j} f_{j+1}\right) \\
& \left.+A_{n+1}^{1, n} f_{n-1}+A_{n+1}^{2, n} f_{n}+A_{n+1}^{3, n} f_{n+1}^{P}\right]
\end{aligned}
$$

where

$$
\begin{aligned}
& A_{n+1}^{1,0}=\frac{2}{h^{2}} \int_{t_{j}}^{t_{j+1}}\left(t_{n+1}-\tau\right)^{\alpha-1}\left(t_{1 / 2}-\tau\right)\left(t_{1}-\tau\right) d \tau, \\
& A_{n+1}^{2,0}=-\frac{4}{h^{2}} \int_{t_{j}}^{t_{j+1}}\left(t_{n+1}-\tau\right)^{\alpha-1}\left(t_{0}-\tau\right)\left(t_{1}-\tau\right) d \tau, \\
& A_{n+1}^{3,0}=\frac{2}{h^{2}} \int_{t_{j}}^{t_{j+1}}\left(t_{n+1}-\tau\right)^{\alpha-1}\left(t_{0}-\tau\right)\left(t_{1 / 2}-\tau\right) d \tau, \\
& A_{n+1}^{1, j}=\frac{1}{2 h^{2}} \int_{t_{j}}^{t_{j+1}}\left(t_{n+1}-\tau\right)^{\alpha-1}\left(t_{j}-\tau\right)\left(t_{j+1}-\tau\right) d \tau, \\
& A_{n+1}^{2, j}=-\frac{1}{h^{2}} \int_{t_{j}}^{t_{j+1}}\left(t_{n+1}-\tau\right)^{\alpha-1}\left(t_{j-1}-\tau\right)\left(t_{j+1}-\tau\right) d \tau,
\end{aligned}
$$




$$
A_{n+1}^{3, j}=\frac{1}{2 h^{2}} \int_{t_{j}}^{t_{j+1}}\left(t_{n+1}-\tau\right)^{\alpha-1}\left(t_{j-1}-\tau\right)\left(t_{j}-\tau\right) d \tau
$$

and the predictor $f_{n+1}^{p}$ is found as follows:

$$
y_{n+1}^{P}=g\left(t_{n+1}\right)+G_{\alpha, f}\left(t_{n+1}\right)+a_{n+1}^{1} f_{n-2}+a_{n+1}^{2} f_{n-1}+a_{n+1}^{3} f_{n},
$$

where

$$
\begin{aligned}
G_{\alpha, f}\left(t_{n+1}\right)= & \frac{1}{\Gamma(\alpha)}\left[A_{n+1}^{1,0} f_{0}+A_{n+1}^{2,0} f_{1 / 2}+A_{n+1}^{3,0} y\left(t_{1}\right)\right. \\
& \left.+\sum_{j=0}^{n-1}\left(A_{n+1}^{1, j} f_{j-1}+A_{n+1}^{2, j} f_{j}+A_{n+1}^{3, j} f_{j+1}\right)\right], \\
2 a_{n+1}^{1}= & \frac{1}{2 h^{2}} \int_{t_{n}}^{t_{n+1}}\left(t_{n+1}-\tau\right)^{\alpha-1}\left(t_{n-1}-\tau\right)\left(t_{n}-\tau\right) d \tau, \\
a_{n+1}^{2}= & -\frac{1}{h^{2}} \int_{t_{n}}^{t_{n+1}}\left(t_{n+1}-\tau\right)^{\alpha-1}\left(t_{n-2}-\tau\right)\left(t_{n}-\tau\right) d \tau, \\
a_{n+1}^{3}= & \frac{1}{2 h^{2}} \int_{t_{n}}^{t_{n+1}}\left(t_{n+1}-\tau\right)^{\alpha-1}\left(t_{n-2}-\tau\right)\left(t_{n-1}-\tau\right) d \tau .
\end{aligned}
$$

Remark 3.1 Using the HPCM with linear and quadratic interpolations, we find predictors $y_{n+1}^{p}, w_{n+1}^{p}, z_{n+1}^{p}$ individually and then find correctors $y_{n+1}^{c}, w_{n+1}^{c}, z_{n+1}^{c}$. The entire steps of the proposed schemes are summarized in Appendix B.

The following theorems [1] bound the global error $E_{n+1}$ of the HPCM with linear and quadratic interpolations, respectively.

Theorem 3.1 (Theorem 2.4 in [1]: Global error of HPCM with linear interpolation) Define $E_{n+1}$ to be global error. Suppose $f(\cdot, y(\cdot)) \in C^{2}[a, b]$ and furthermore is Lipschitz continuous in the second argument, then we have

$$
E_{n+1}=\left|y\left(t_{n+1}\right)-\tilde{y}_{n+1}\right| \leq \mathcal{O}\left(h^{2}\right),
$$

given $E_{1} \leq C h^{2}$.

Theorem 3.2 (Theorem 3.4 in [1]: Global error of HPCM with quadratic interpolation) Suppose $f(\cdot, y(\cdot)) \in C^{3}[a, b]$ and is Lipschitz continuous in the second argument, then we have

$$
E_{n+1} \leq \mathcal{O}\left(h^{3}\right),
$$

given $E_{1}, E_{2} \leq \mathcal{O}\left(h^{3}\right)$ and $E_{1 / 2} \leq O\left(h^{3-\alpha}\right), 0<\alpha<1$

\section{Numerical examples}

In this section, we experimentally illustrate the performance of the proposed schemes. Numerically, we verify that our proposed schemes can deal with more complex FBVPs than 
the integral discretization schemes in $[20,21]$. For that purpose, the proposed schemes are implemented in FNBVPs with $0<\alpha_{1}<1$ whose exact solutions are polynomial, exponential, and sine functions in Examples 4.1 through 4.3. We investigate absolute errors in maximum norm, convergence rates, and absolute values of the approximated error function $\left|\tilde{F}\left(s_{k}\right)\right|$ with various values of parameters. We discuss linear FBVPs with $\alpha_{1}=1$ whose exact solutions have low regularity and high regularity in Examples 4.4 and 4.5, respectively. We compare numerical results obtained by our proposed schemes with the integral discretization schemes. But we emphasize that our proposed methods can deal with many different FBVPs unlike the another method in Examples 4.4 and 4.5. Regarding the numerical results shown in the Appendix, let us summarize the parameters used:

- $h$ denotes the size of time sub-interval.

- $s_{0}$ denotes the initial approximation of the sequence $\left\{s_{k}\right\}$ in proposed shooting methods.

- $k$ denotes the number of iterations needed to meet a tolerance in the sequence $\left\{s_{k}\right\}$ generated by the proposed Newton's method or Halley's method.

- $m$ denotes the maximum number of iterations in Newton's and Halley's methods.

- $T o l$ denotes the tolerance used to measure the error of the approximated error function $\left|\tilde{F}\left(s_{k}\right)\right|$ in Newton's method and Halley's method.

- $N$ denotes the number of time sub-intervals.

- $y_{j}^{c}$ denotes the approximate solution at $t_{j}$ obtained by the proposed shooting technique based on either Newton's method or Halley's method, with HPCM. $y\left(t_{j}\right)$ denotes the exact solution at $t_{j}$.

- Max. error denotes the pointwise absolute errors in the maximum norm. (i.e. $\left.\max _{1 \leq j \leq N}\left|y_{j}^{c}-y\left(t_{j}\right)\right|\right)$

- $E_{\alpha, \beta}(t)$ denotes the two-parameter function of Mittag-Leffler type [7].

In Examples 4.1 through 4.3, we transform the FNBVP into the system of FIVPs (7) and $s_{0}$ means an initial approximation to $y^{\prime}(0)$. In Examples 4.4 and 4.5, the linear FBVP is transformed into the system of FIVPs (22) with $\epsilon=10^{-10}$ and $s_{0}$ means an initial approximation to $y(0)$. For all examples except for Example 4.4, we implement the shooting technique based on Newton's method (Halley's method) combined with HPCM with linear (quadratic) interpolation to verify the order of convergence $\mathcal{O}\left(h^{2}\right)\left(\mathcal{O}\left(h^{3}\right)\right)$, respectively.

Example 4.1 Consider the following double-term FNBVP with RBCs:

$$
\left\{\begin{array}{l}
D_{0}^{\alpha_{2}} y(t)=\frac{\Gamma(5)}{\Gamma\left(5-\alpha_{2}\right)} t^{4-\alpha_{2}}-\frac{\Gamma(5)}{\Gamma\left(5-\alpha_{1}\right)} t^{4-\alpha_{1}}-t^{8}+y^{2}+D_{0}^{\alpha_{1}} y(t), \\
y(0)+y^{\prime}(0)=0, \quad y(1)+y^{\prime}(1)=5,
\end{array}\right.
$$

where the exact solution is $y(t)=t^{4}$.

Example 4.2 Consider the following double-term FNBVP with RBCs:

$$
\left\{\begin{array}{l}
D_{0}^{\alpha_{2}} y(t)=\lambda^{2} t^{2-\alpha_{2}} E_{1,3-\alpha_{2}}(\lambda t)-\left(\lambda^{2} \frac{\Gamma(3)}{2 \Gamma\left(3-\alpha_{2}\right)} t^{2-\alpha_{2}}+\lambda^{3} \frac{\Gamma(4)}{6 \Gamma\left(4-\alpha_{2}\right)} t^{3-\alpha_{2}}\right) \\
\quad-A^{2}+y^{2}-t B+t D_{0}^{\alpha_{1}} y(t), \\
y(0)+y^{\prime}(0)=0, \quad y(1)+y^{\prime}(1) \approx 0.2699
\end{array}\right.
$$


where

$$
\begin{aligned}
\lambda= & 1, \\
A= & e^{\lambda t}-\left(1+\lambda t+\frac{\lambda^{2}}{2} t^{2}+\frac{\lambda^{3}}{3 !} t^{3}\right), \\
B= & \lambda t^{1-\alpha_{1}} E_{1,2-\alpha_{1}}(\lambda t) \\
& -\left(\lambda \frac{\Gamma(2)}{\Gamma\left(2-\alpha_{1}\right)} t^{1-\alpha_{1}}+\lambda^{2} \frac{\Gamma(3)}{2 \Gamma\left(3-\alpha_{1}\right)} t^{2-\alpha_{1}}+\lambda^{3} \frac{\Gamma(4)}{6 \Gamma\left(4-\alpha_{1}\right)} t^{3-\alpha_{1}}\right),
\end{aligned}
$$

and the exact solution is $y(t)=e^{\lambda t}-\left(1+\lambda t+\frac{\lambda^{2}}{2} t^{2}+\frac{\lambda^{3}}{3 !} t^{3}\right)$.

Example 4.3 Consider the following double-term FNBVP with RBCs:

$$
\left\{\begin{array}{c}
D_{0}^{\alpha_{2}} y(t)=F_{\lambda}^{\left(\alpha_{2}\right)}(t)+\frac{\Gamma(4)}{6 \Gamma\left(4-\alpha_{2}\right)} t^{3-\alpha_{2}}+\left(\sin (t)-t+\frac{t^{3}}{6}\right)^{2}-y^{2} \\
\quad-F_{\lambda}^{\left(\alpha_{1}\right)}(t)+\frac{\Gamma(2)}{\Gamma\left(2-\alpha_{1}\right)} t^{1-\alpha_{1}}-\frac{\Gamma(4)}{6 \Gamma\left(4-\alpha_{1}\right)} t^{3-\alpha_{1}}+D_{0}^{\alpha_{1}} y(t), \\
y(0)+y^{\prime}(0)=0, \quad y(1)+y^{\prime}(1) \approx 4.84399,
\end{array}\right.
$$

where

$$
\begin{aligned}
F_{\lambda}^{(\alpha)}(t)= & -\frac{1}{2} i(i \lambda)^{(\lceil\alpha\rceil)} t^{[\lceil\alpha\rceil-\alpha)} \\
& \cdot\left[E_{\lceil\alpha\rceil-\alpha+1}(i \lambda t)-(-1)^{(\lceil\alpha])} E_{1,\lceil\alpha]-\alpha+1}(-i \lambda t)\right], \\
\lambda=1, &
\end{aligned}
$$

and the exact solution is $y(t)=\sin (\lambda t)-t+\frac{t^{3}}{6}$.

\begin{tabular}{|c|c|c|c|c|c|c|c|c|c|c|}
\hline \multirow[t]{2}{*}{$m$} & \multicolumn{5}{|c|}{ Newton's method } & \multicolumn{5}{|c|}{ Halley's method } \\
\hline & $s_{0}=0.2$ & $s_{0}=0.4$ & $s_{0}=0.6$ & $s_{0}=0.8$ & $s_{0}=1.0$ & $\overline{s_{0}=0.2}$ & $s_{0}=0.4$ & $s_{0}=0.6$ & $s_{0}=0.8$ & $s_{0}=1.0$ \\
\hline 1 & 0.604011 & 1.305541 & 2.105771 & 3.00776 & 4.017051 & 0.603191 & 1.304704 & 2.104838 & 3.006644 & 4.01565 \\
\hline 2 & 0.042234 & $1.48 \mathrm{E}-01$ & 0.303846 & 5.05E-01 & 0.753495 & 0.054329 & $1.90 \mathrm{E}-01$ & 0.392297 & $6.59 \mathrm{E}-01$ & 0.995211 \\
\hline 3 & 0.000274 & $3.25 \mathrm{E}-03$ & 0.01249 & 3.10E-02 & 0.061341 & 0.000604 & $6.84 \mathrm{E}-03$ & 0.025715 & $6.31 \mathrm{E}-02$ & 0.124161 \\
\hline 4 & 7.31E-08 & 7.33E-07 & $2.17 \mathrm{E}-05$ & $1.46 \mathrm{E}-04$ & 0.000578 & $9.11 \mathrm{E}-08$ & $8.24 \mathrm{E}-06$ & $1.34 \mathrm{E}-04$ & 0.000813 & 0.003036 \\
\hline 5 & $2.28 \mathrm{E}-11$ & $2.29 E-10$ & $6.71 \mathrm{E}-09$ & $4.22 \mathrm{E}-08$ & $1.25 \mathrm{E}-07$ & $2.58 \mathrm{E}-11$ & $2.32 \mathrm{E}-09$ & $3.41 \mathrm{E}-08$ & 8.56E-08 & $1.15 E-06$ \\
\hline 6 & $7.11 \mathrm{E}-15$ & 7.19E-14 & $2.10 \mathrm{E}-12$ & $1.32 \mathrm{E}-11$ & $3.91 \mathrm{E}-11$ & $6.22 \mathrm{E}-15$ & $6.55 E-13$ & $9.64 \mathrm{E}-12$ & $2.42 \mathrm{E}-11$ & $3.26 \mathrm{E}-10$ \\
\hline 7 & 0 & 0 & $8.88 \mathrm{E}-16$ & 4.44E-15 & $1.07 E-14$ & $8.88 \mathrm{E}-16$ & 0 & $2.66 \mathrm{E}-15$ & $6.22 \mathrm{E}-15$ & $9.15 E-14$ \\
\hline 8 & 0 & 0 & 0 & 0 & $1.78 \mathrm{E}-15$ & $8.88 \mathrm{E}-16$ & 0 & $8.88 \mathrm{E}-16$ & 0 & $8.88 \mathrm{E}-16$ \\
\hline 9 & 0 & 0 & 0 & 0 & 0 & $8.88 \mathrm{E}-16$ & 0 & $8.88 \mathrm{E}-16$ & 0 & $8.88 \mathrm{E}-16$ \\
\hline 10 & 0 & 0 & 0 & 0 & 0 & $8.88 \mathrm{E}-16$ & 0 & $8.88 \mathrm{E}-16$ & 0 & $8.88 \mathrm{E}-16$ \\
\hline
\end{tabular}

In Examples 4.1, 4.2, and 4.3, we observe the following:

1 For all three examples, $w(t), z(t), f(t, y, w)$ (e.g. $\left.D_{0}^{\alpha_{1}} y(t), y^{\prime}(t), f\left(t, y, D_{0}^{\alpha_{2}} y(t)\right)\right)$ belong to $C^{3}[0,1]$. By Theorems 3.1 and 3.2, thus, computed convergence profiles are estimated $\mathcal{O}\left(h^{2}\right)$ and $\mathcal{O}\left(h^{3}\right)$ for the HPCM with linear (quadratic) interpolation combined with shooting technique based on Newton's (Halley's) method, respectively.

Table 1 Errors of shooting techniques $\left|\tilde{F}\left(s_{m}\right)\right|$ in (41) versus the maximum number of iterations with various values of $s_{0}$ in Example 4.1. We set $h=0.01, \alpha_{1}=0.4, \alpha_{2}=1.7$ 
Table 2 Pointwise absolute errors in the maximum norm and computed rates of convergence versus the number of sub-intervals $N$ with $s_{0}=0.2,1.0$ for each in Example 4.1. We set $\alpha_{1}=0.4$, $\alpha_{2}=1.7$, and $m$ is fixed at 10

\begin{tabular}{|c|c|c|c|c|c|c|c|c|}
\hline \multirow[t]{3}{*}{ N } & \multicolumn{4}{|c|}{ Newton's method } & \multicolumn{4}{|c|}{ Halley's method } \\
\hline & \multicolumn{2}{|l|}{$s_{0}=0.2$} & \multicolumn{2}{|l|}{$s_{0}=1.0$} & \multicolumn{2}{|l|}{$s_{0}=0.2$} & \multicolumn{2}{|l|}{$s_{0}=1.0$} \\
\hline & Max. error & Rate & Max. error & Rate & Max. error & Rate & Max. error & Rate \\
\hline 10 & $1.17 \mathrm{E}-02$ & - & $1.17 \mathrm{E}-02$ & - & $1.02 \mathrm{E}-03$ & - & $1.02 \mathrm{E}-03$ & - \\
\hline 20 & $5.63 \mathrm{E}-03$ & 1.059 & $5.63 \mathrm{E}-03$ & 1.059 & $1.83 \mathrm{E}-04$ & 2.486 & $1.83 \mathrm{E}-04$ & 2.486 \\
\hline 40 & $1.78 \mathrm{E}-03$ & 1.665 & $1.78 \mathrm{E}-03$ & 1.665 & $3.63 \mathrm{E}-05$ & 2.333 & $3.63 \mathrm{E}-05$ & 2.332 \\
\hline 80 & $4.92 \mathrm{E}-04$ & 1.855 & $4.92 \mathrm{E}-04$ & 1.854 & 5.47E-06 & 2.730 & 5.47E-06 & 2.730 \\
\hline 160 & $1.29 \mathrm{E}-04$ & 1.931 & $1.29 \mathrm{E}-04$ & 1.931 & $7.51 \mathrm{E}-07$ & 2.866 & $7.51 \mathrm{E}-07$ & 2.866 \\
\hline 320 & 3.30E-05 & 1.966 & 3.30E-05 & 1.966 & 9.88E-08 & 2.925 & $9.88 \mathrm{E}-08$ & 2.925 \\
\hline
\end{tabular}

2 Tables 1, 4, 7 show the absolute values of approximated error function (41) at $s_{m}$ (i.e. $\left.\left|a_{2} y_{N}^{c}\left(s_{m}\right)+b_{2} z_{N}^{c}\left(s_{m}\right)-\gamma_{2}\right|\right)$ versus the maximum number of iterations $m$ with various initial values $s_{0} . y_{N}^{c}\left(s_{m}\right)$ and $z_{N}^{c}\left(s_{m}\right)$ are computed by using proposed schemes. We set $\alpha_{1}=0.4, \alpha_{2}=1.7, h=0.01$ in all tables. From numerical results in those tables, we can verify that the sequence $\left\{s_{k}\right\}$ obtained by the proposed shooting algorithms approaches to the IC $s$ within the error at least $10^{-16}$ when $m$ is at most 10 with $s_{0}=0.2,0.4,0.6,0.8,1.0$ for each. This leads us to the conclusion that the proposed shooting techniques show a good performance with remarkable accuracy regarding to approximation of the IC $s$.

3 Tables 2, 5, 8 show pointwise absolute errors in the maximum norm and convergence rates computed versus the number of sub-intervals $N$ in the cases of $s_{0}=0.2,1.0$ for each of Newton's and Halley's method. We set $\alpha_{1}=0.4, \alpha_{2}=1.7$. The sequence $\left\{s_{k}\right\}$ was computed up to $s_{10}$ so, based on the observation of Tables $1,4,7$, we see that the error of the approximated error function $\left|\tilde{F}\left(s_{k}\right)\right|$ does not have an effect on the convergence rate of $y_{j}^{c}$ obtained by HPCMs. In Tables 2, 5, 8, we can see that computed convergence profiles obtained by the proposed schemes approach 2 for Newton's method and 3 for Halley's method as $N$ is increased. Thus numerical results shown in those tables support that the proposed methods follow global error estimates of HPCMs.

4. Proposed methods are tested for a variety of values of $\alpha_{1}, \alpha_{2}$ and numerical results for each pair of $\left(\alpha_{1}, \alpha_{2}\right)$ are shown in Tables 3, 6, 9. For each pair of fractional orders $\left(\alpha_{1}, \alpha_{2}\right)$ pointwise absolute errors in the maximum norm, computed convergence rates, CPU time executed in seconds, and number of iterations $k$ such that $\left|\tilde{F}\left(s_{k}\right)\right|<T o l$ versus the number of sub-intervals $N$ are listed in the tables. The initial approximation to $s$ was set $s_{0}=0.2$ in all three tables. In order to minimize the number of iterations $k$, the tolerance was set $T o l=10^{-5}$ for Newton's method and $T o l=10^{-10}$ for Halley's method in Table 3, $T o l=10^{-10}$ for both shooting techniques in Table 6, and $T o l=10^{-15}$ for Newton's method and $T o l=10^{-16}$ for Halley's method in Table 9. Numerical results shown in the tables demonstrate that, for all suggested pairs of fractional orders, rates of convergence approach 2 for Newton's method, 3 for Halley's method that are theoretical convergence rates of HPCMs. In Tables 3 and 9, we observe that the number of iterations $k$ required to meet the tolerance at $(0.9,1.1)$ is relatively greater than other pairs of fractional orders for both Newton's and Halley's method. 
Kim et al. Advances in Difference Equations

(2021) 2021:193

Page 18 of 35

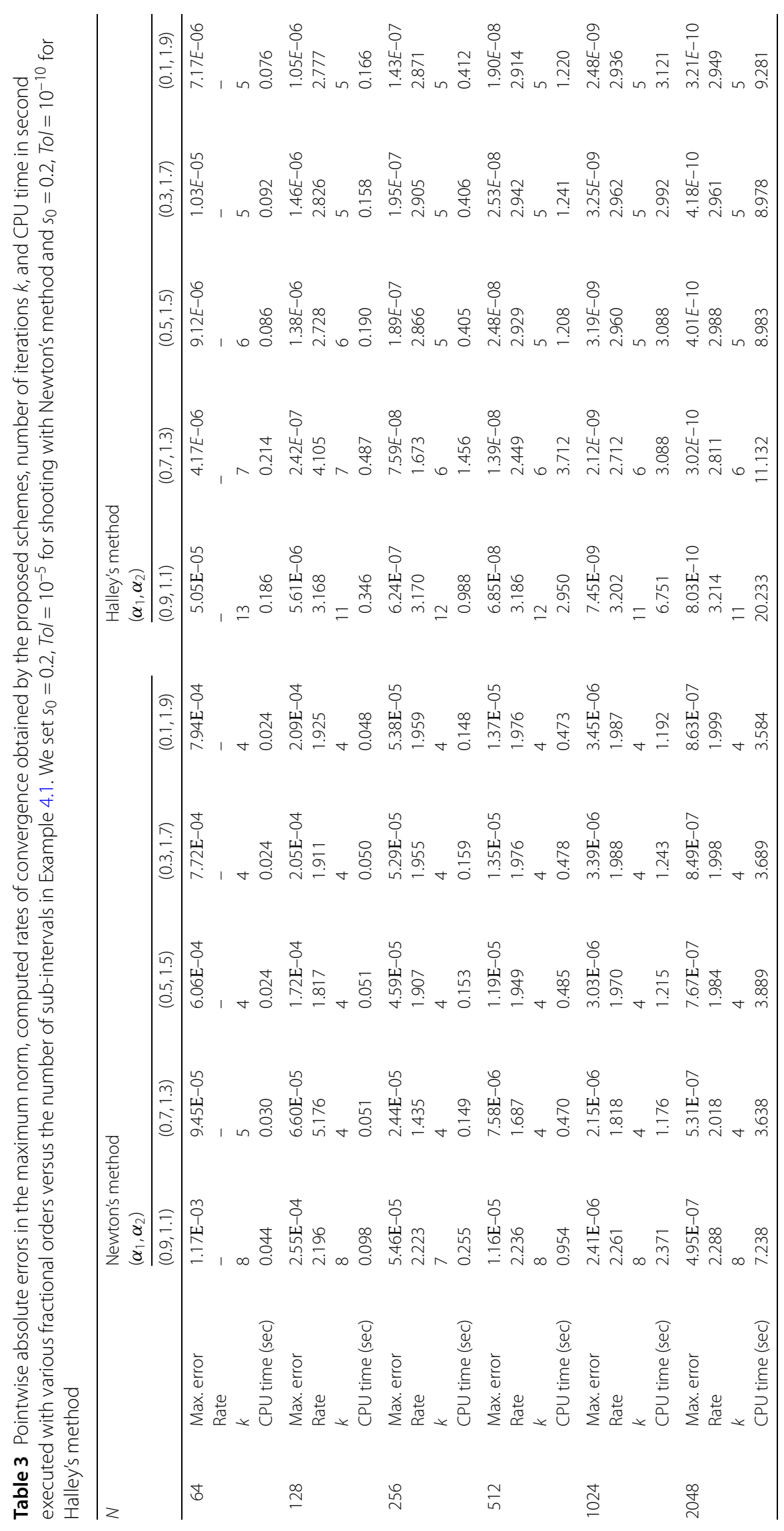


Table 4 Errors of shooting techniques $\left|\tilde{F}\left(s_{m}\right)\right|$ in (41) versus the maximum number of iterations $m$ for each $s_{0}$ in Example 4.2 as we set $\alpha_{1}=0.4, \alpha_{2}=1.7, h=0.01$

\begin{tabular}{|c|c|c|c|c|c|c|c|c|c|c|}
\hline \multirow[t]{2}{*}{$m$} & \multicolumn{5}{|c|}{ Newton's method } & \multicolumn{5}{|c|}{ Halley's method } \\
\hline & $s_{0}=0.2$ & $s_{0}=0.4$ & $s_{0}=0.6$ & $s_{0}=0.8$ & $s_{0}=1.0$ & $s_{0}=0.2$ & $s_{0}=0.4$ & $s_{0}=0.6$ & $s_{0}=0.8$ & $s_{0}=1.0$ \\
\hline 1 & 0.414113 & 0.890547 & 1.42639 & 2.02066 & 2.674035 & 0.414046 & 0.890413 & 1.426145 & 2.020256 & 2.673415 \\
\hline 2 & 0.027748 & $9.51 \mathrm{E}-02$ & 0.190648 & $3.11 \mathrm{E}-01$ & 0.456212 & 0.03142 & $1.08 \mathrm{E}-01$ & 0.219523 & $3.66 \mathrm{E}-01$ & 0.549419 \\
\hline 3 & 0.000177 & $1.93 \mathrm{E}-03$ & 0.007068 & 1.70E-02 & 0.032652 & 0.000258 & $2.80 \mathrm{E}-03$ & 0.010391 & $2.54 \mathrm{E}-02$ & 0.050225 \\
\hline 4 & $1.51 \mathrm{E}-08$ & $9.56 \mathrm{E}-07$ & 1.20E-05 & $6.71 \mathrm{E}-05$ & 0.000243 & 1.61E-08 & $2.10 \mathrm{E}-06$ & $2.89 \mathrm{E}-05$ & 1.70E-04 & 0.000646 \\
\hline 5 & $6.59 \mathrm{E}-13$ & $4.20 \mathrm{E}-11$ & $5.57 \mathrm{E}-10$ & 3.99E-09 & $2.45 \mathrm{E}-08$ & $1.25 \mathrm{E}-13$ & $1.52 \mathrm{E}-11$ & $1.33 \mathrm{E}-12$ & 6.56E-09 & $1.08 \mathrm{E}-07$ \\
\hline 6 & 0 & 1.89E-15 & $2.44 \mathrm{E}-14$ & $1.75 E-13$ & $1.07 \mathrm{E}-12$ & $5.55 E-17$ & $1.67 \mathrm{E}-16$ & $1.11 \mathrm{E}-16$ & $5.11 \mathrm{E}-14$ & $8.40 E-13$ \\
\hline 7 & 0 & 0 & 0 & 0 & $1.67 \mathrm{E}-16$ & $5.55 \mathrm{E}-17$ & $5.55 E-17$ & $5.55 \mathrm{E}-17$ & $5.55 \mathrm{E}-17$ & $5.55 E-17$ \\
\hline 8 & 0 & 0 & 0 & 0 & $5.55 \mathrm{E}-17$ & $5.55 \mathrm{E}-17$ & $5.55 \mathrm{E}-17$ & $5.55 \mathrm{E}-17$ & $5.55 \mathrm{E}-17$ & 0 \\
\hline 9 & 0 & 0 & 0 & 0 & 0 & $5.55 \mathrm{E}-17$ & $5.55 \mathrm{E}-17$ & $5.55 \mathrm{E}-17$ & $5.55 \mathrm{E}-17$ & 0 \\
\hline 10 & 0 & 0 & 0 & 0 & 0 & $5.55 \mathrm{E}-17$ & $5.55 \mathrm{E}-17$ & $5.55 \mathrm{E}-17$ & $5.55 \mathrm{E}-17$ & 0 \\
\hline
\end{tabular}

Table 5 Pointwise absolute errors in the maximum norm and computed rates of convergence versus the number of sub-intervals $N$ in Example 4.2 as we set $\alpha_{1}=0.4, \alpha_{2}=1.7, h=0.01, m=10$ and no Tol

\begin{tabular}{|c|c|c|c|c|c|c|c|c|}
\hline \multirow[t]{3}{*}{$\bar{N}$} & \multicolumn{4}{|c|}{ Newton's method } & \multicolumn{4}{|c|}{ Halley's method } \\
\hline & \multicolumn{2}{|l|}{$s_{0}=0.2$} & \multicolumn{2}{|l|}{$s_{0}=1.0$} & \multicolumn{2}{|l|}{$s_{0}=0.2$} & \multicolumn{2}{|l|}{$s_{0}=1.0$} \\
\hline & $\overline{\text { Max. error }}$ & $\overline{\text { Rate }}$ & Max. error & $\overline{\text { Rate }}$ & $\overline{\text { Max. error }}$ & $\overline{\text { Rate }}$ & Max. error & Rate \\
\hline 10 & $1.10 \mathrm{E}-03$ & - & $1.10 \mathrm{E}-03$ & - & $5.65 \mathrm{E}-05$ & - & $5.65 \mathrm{E}-05$ & - \\
\hline 20 & $4.05 E-04$ & 1.434 & 4.05E-04 & 1.434 & $1.71 \mathrm{E}-05$ & 1.725 & $1.71 \mathrm{E}-05$ & 1.725 \\
\hline 40 & $1.19 \mathrm{E}-04$ & 1.765 & 1.19E-04 & 1.765 & $2.82 \mathrm{E}-06$ & 2.599 & $2.82 \mathrm{E}-06$ & 2.599 \\
\hline 80 & $3.21 \mathrm{E}-05$ & 1.893 & $3.21 \mathrm{E}-05$ & 1.893 & 3.99E-07 & 2.824 & 3.99E-07 & 2.824 \\
\hline 160 & $8.32 \mathrm{E}-06$ & 1.949 & $8.32 \mathrm{E}-06$ & 1.949 & $5.29 \mathrm{E}-08$ & 2.913 & $5.29 \mathrm{E}-08$ & 2.913 \\
\hline 320 & $2.12 \mathrm{E}-06$ & 1.974 & $2.12 \mathrm{E}-06$ & 1.974 & $6.84 \mathrm{E}-09$ & 2.952 & $6.84 \mathrm{E}-09$ & 2.952 \\
\hline
\end{tabular}

5 Figures 1, 2, and 3 illustrate convergence profiles obtained by the proposed methods with the variety of fractional orders. From the figures, we can see that computed rates of convergence are nearly $\mathcal{O}\left(h^{2}\right)$ and $\mathcal{O}\left(h^{3}\right)$ for the proposed shooting method with Newton's and Halley's schemes, respectively. Figures 4 and 5 display graphs of pointwise absolute errors versus the time step $h$. The approximate solution is computed by the proposed method with Newton's method (in Fig. 4) and Halley's method (in Fig. 5), respectively.

Example 4.4 Consider the following single-term linear FBVP with RBCs:

$$
\left\{\begin{array}{l}
D_{0}^{\alpha_{2}} y(t)=\varphi(t)-(2 t+6) y^{\prime}(t) \\
y(0)-\frac{1}{1-\alpha_{2}} y^{\prime}(0)=\gamma_{1}, \quad y(1)+y^{\prime}(1)=\gamma_{2},
\end{array}\right.
$$

where $1<\alpha_{2}<2$,

$$
\begin{aligned}
\varphi(t)= & \frac{\Gamma\left(\alpha_{2}+1\right)}{\Gamma(1)}+\frac{\Gamma\left(2 \alpha_{2}\right)}{\Gamma\left(\alpha_{2}\right)} t^{\left(\alpha_{2}-1\right)}+4 \frac{\Gamma(4)}{\Gamma\left(4-\alpha_{2}\right)} t^{\left(3-\alpha_{2}\right)}+\frac{\Gamma(5)}{\Gamma\left(5-\alpha_{2}\right)} t^{\left(4-\alpha_{2}\right)} \\
& +(2 t+6)\left(\alpha_{2} t^{\alpha_{2}-1}+\left(2 \alpha_{2}-1\right) t^{2 \alpha_{2}-2}+3+12 t^{2}+4 t^{3}\right),
\end{aligned}
$$

and the exact solution is $y(t)=t^{\alpha_{2}}+t^{2 \alpha_{2}-1}+1+3 t+4 t^{3}+t^{4}[21]$.

Since $D_{0}^{\alpha_{1}} y(t), y^{\prime}(t), D_{0}^{\alpha_{2}} y(t)$ do not belong to $C^{3}[0,1]$, global error estimates of HPCMs in Theorems 3.1 and 3.2 cannot be applied to Example 4.4. Alternatively, we adopt the linear explicit method described in Appendix A with proposed shooting techniques. In this 


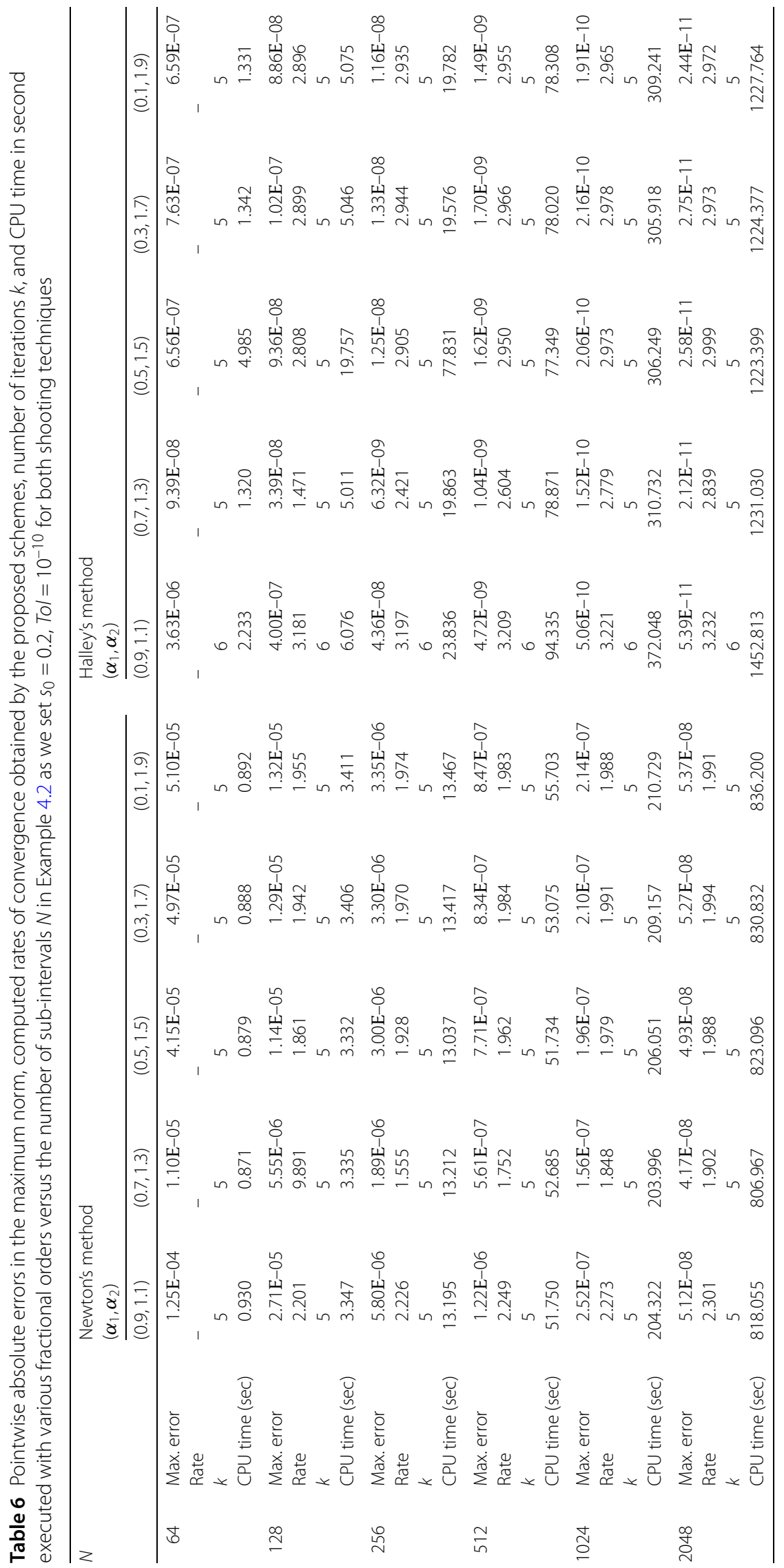


Table 7 Errors of shooting techniques $\left|\tilde{F}\left(s_{m}\right)\right|$ in (41) versus the maximum number of iterations $m$ with various initial approximations $s_{0}$ in Example 4.3 as we set $\alpha_{1}=0.4, \alpha_{2}=1.7, h=0.01$

\begin{tabular}{|c|c|c|c|c|c|c|c|c|c|c|}
\hline \multirow[t]{2}{*}{$m$} & \multicolumn{5}{|c|}{ Newton's method } & \multicolumn{5}{|c|}{ Halley's method } \\
\hline & $s_{0}=0.2$ & $s_{0}=0.4$ & $s_{0}=0.6$ & $s_{0}=0.8$ & $s_{0}=1.0$ & $s_{0}=0.2$ & $s_{0}=0.4$ & $s_{0}=0.6$ & $s_{0}=0.8$ & $s_{0}=1.0$ \\
\hline 1 & 0.487617 & 0.891569 & 1.202993 & 1.407814 & 1.485109 & 0.487722 & 0.891834 & 1.203474 & 1.408578 & 1.486244 \\
\hline 2 & 1684 & $3.44 \mathrm{E}-01$ & 0.653431 & 9.7 & 05 & 65 & & 0.6 & 9.9 & 771 \\
\hline 3 & 0.004491 & 5.07E-02 & 0.182979 & 4.1 & 0.7 & 85 & & 315 & -01 & 0.757881 \\
\hline 4 & $1.26 \mathrm{E}-05$ & 1.14E-03 & 0.014409 & 0.073158 & 0.226095 & 05 & 1.5 & $1.80 \mathrm{E}-02$ & -02 & 0.255888 \\
\hline 5 & $1.13 \mathrm{E}-08$ & $1.57 \mathrm{E}-06$ & 0.000101 & 0.002343 & 0.021949 & 1.5 & & -04 & -03 & 2.96 \\
\hline 6 & $1.01 \mathrm{E}-11$ & $1.41 \mathrm{E}-09$ & $9.49 \mathrm{E}-08$ & 4.43E-06 & 0.000225 & $1.39 \mathrm{E}-11$ & $2.16 \mathrm{E}-09$ & $1.59 \mathrm{E}-07$ & $8.62 \mathrm{E}-06$ & $4.22 \mathrm{E}-04$ \\
\hline 7 & $9.06 \mathrm{E}-15$ & $1.26 \mathrm{E}-12$ & $8.48 \mathrm{E}-11$ & 3.97E-09 & $2.22 \mathrm{E}-07$ & $1.25 \mathrm{E}-14$ & $1.95 \mathrm{E}-12$ & $1.44 \mathrm{E}-10$ & 7.82E-09 & $4.62 \mathrm{E}-07$ \\
\hline 8 & $6.94 \mathrm{E}-18$ & $1.12 \mathrm{E}-15$ & $7.58 \mathrm{E}-14$ & $3.55 \mathrm{E}-12$ & $1.99 \mathrm{E}-10$ & $6.94 \mathrm{E}-18$ & $1.76 E-15$ & $1.30 E-13$ & $7.06 \mathrm{E}-12$ & $4.17 \mathrm{E}-10$ \\
\hline 9 & 0 & $1.39 \mathrm{E}-17$ & $6.94 \mathrm{E}-17$ & $3.16 \mathrm{E}-15$ & $1.78 \mathrm{E}-13$ & $6.94 \mathrm{E}-18$ & $6.94 \mathrm{E}-18$ & $1.25 \mathrm{E}-16$ & $6.37 \mathrm{E}-15$ & $3.77 \mathrm{E}$ \\
\hline 10 & 0 & $6.94 \mathrm{E}-18$ & $6.94 \mathrm{E}-18$ & $6.94 \mathrm{E}-18$ & $1.67 \mathrm{E}-16$ & $6.94 \mathrm{E}-18$ & 0 & $6.94 \mathrm{E}-18$ & $6.94 \mathrm{E}-18$ & $3.33 \mathrm{E}-16$ \\
\hline
\end{tabular}

Table 8 Pointwise absolute errors in the maximum norm and computed rates of convergence versus the number of sub-intervals $N$ in Example 4.3 as we set $\alpha_{1}=0.4, \alpha_{2}=1.7, m=10$

\begin{tabular}{|c|c|c|c|c|c|c|c|c|}
\hline \multirow[t]{3}{*}{$N$} & \multicolumn{4}{|c|}{ Newton's method } & \multicolumn{4}{|c|}{ Halley's method } \\
\hline & \multicolumn{2}{|l|}{$s_{0}=0.2$} & \multicolumn{2}{|l|}{$s_{0}=1.0$} & \multicolumn{2}{|l|}{$s_{0}=0.2$} & \multicolumn{2}{|l|}{$s_{0}=1.0$} \\
\hline & Max. error & Rate & Max. error & Rate & Max. error & Rate & Max. error & Rate \\
\hline 10 & $1.73 \mathrm{E}-04$ & - & $1.73 \mathrm{E}-04$ & - & 1.19E-05 & - & 1.19E-05 & - \\
\hline 20 & $6.85 \mathrm{E}-05$ & 1.338 & $6.85 \mathrm{E}-05$ & 1.338 & $3.97 \mathrm{E}-06$ & 1.578 & $3.97 \mathrm{E}-06$ & 1.578 \\
\hline 40 & 2.07E-05 & 1.727 & 2.07E-05 & 1.727 & $6.62 \mathrm{E}-07$ & 2.586 & $6.62 \mathrm{E}-07$ & 2.586 \\
\hline 80 & $5.63 \mathrm{E}-06$ & 1.877 & $5.63 \mathrm{E}-06$ & 1.877 & $9.28 \mathrm{E}-08$ & 2.834 & $9.28 \mathrm{E}-08$ & 2.834 \\
\hline 160 & 1.47E-06 & 1.942 & 1.47E-06 & 1.942 & $1.22 \mathrm{E}-08$ & 2.926 & $1.22 \mathrm{E}-08$ & 2.926 \\
\hline 320 & $3.74 \mathrm{E}-07$ & 1.971 & $3.74 \mathrm{E}-07$ & 1.971 & $1.56 \mathrm{E}-09$ & 2.966 & $1.56 \mathrm{E}-09$ & 2.966 \\
\hline
\end{tabular}

example, we compare the accuracy and convergence rate of the approximate solution obtained by the proposed shooting technique based on Newton's method with the modified integral discretization scheme [21] for each $\alpha_{2}=1.1,1.3,1.5,1.7,1.9$. In Table 10, we can observe that our proposed method consumes less CPU time than the modified integral discretization scheme [21] even though both methods shows the equal performance.

Example 4.5 Consider the following single-term linear FBVP with RBCs:

$$
\left\{\begin{array}{l}
D_{0}^{\alpha_{2}} y(t)=F(t)-\cos (t) y(t)-\sin (t) y^{\prime}(t) \\
y(0)-\frac{1}{1-\alpha_{2}} y^{\prime}(0)=\gamma_{1}, \quad y(1)+y^{\prime}(1)=\gamma_{2}
\end{array}\right.
$$

whose $1<\alpha<2$ and the exact solution is

$$
y(t)=\sin (\lambda t)-t+\frac{t^{3}}{6}
$$

In Example 4.5, we compare the performance of our proposed methods with the modified integral discretization scheme [21]. Table 11 shows pointwise absolute errors and computed convergence profiles versus the number of sub-intervals for each $\alpha_{2}=$ $1.1,1.3,1.5,1.7,1.9$. In Table 11 , we can see that, for all values of $\alpha_{2}$, the computed rates of convergence obtained by the proposed shooting technique based on Halley's method combined with third-order HPCM are around 3.0 while the computed rates of convergence obtained by the modified integral discretization scheme [21] are around 2.0. 
Kim et al. Advances in Difference Equations

(2021) 2021:193

Page 22 of 35

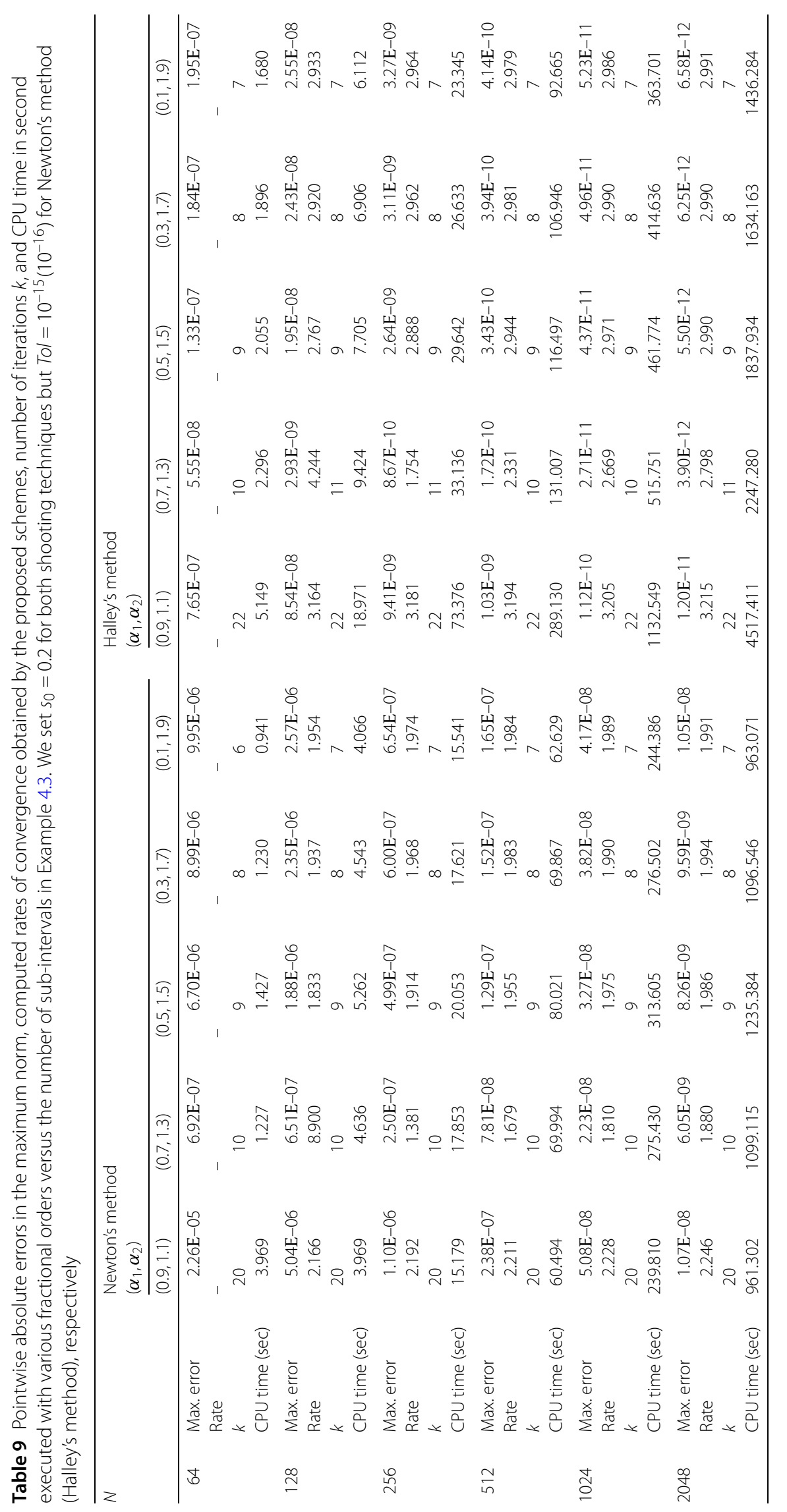




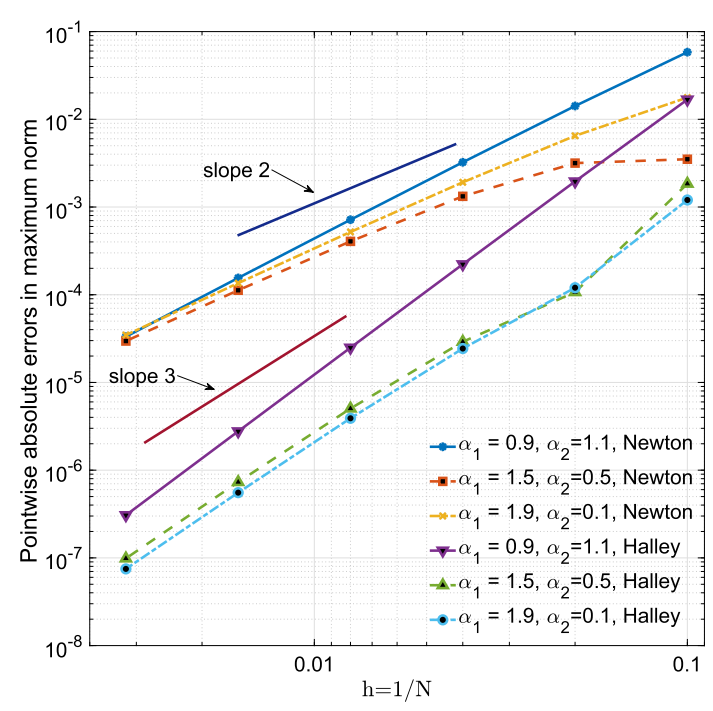

Figure 1 Pointwise absolute errors in maximum norm of Example 4.1 versus the time step $h$ when $\left(\alpha_{1}, \alpha_{2}\right)=(0.9,1.1),(1.5,0.5),(1.9,0.1)$. Graphs demonstrate that computed convergence profiles are nearly $\mathcal{O}\left(h^{2}\right)$ and $\mathcal{O}\left(h^{3}\right)$ for Newton's and Halley's method, respectively

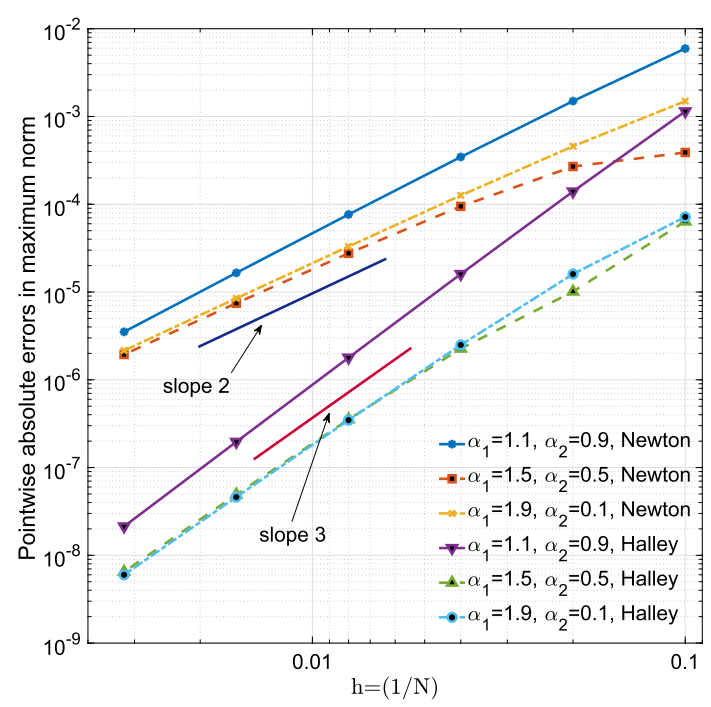

Figure 2 Pointwise absolute errors in maximum norm of Example 4.2 versus the time step $h$ when $\left(\alpha_{1}, \alpha_{2}\right)=(0.9,1.1),(1.5,0.5),(1.9,0.1)$. Graphs demonstrate that computed convergence profiles are nearly $\mathcal{O}\left(h^{2}\right)$ and $\mathcal{O}\left(h^{3}\right)$ for Newton's and Halley's method, respectively

The algorithm of the proposed shooting techniques with second-order HPCM requires less than the number of arithmetic operations needed by the modified integral discretization scheme to solve a FBVP with RBCs than the modified integral discretization scheme [21] because the predictor and corrector in HPCMs share the computation of the memory effect. As a result the proposed shooting technique based on Newton's method consumes less CPU than the modified discretization scheme [21] and the CPU time executed by the proposed shooting technique based on Halley's method is approximately equal to the CPU time executed by the modified integral discretization scheme [21], as shown in Table 11. 


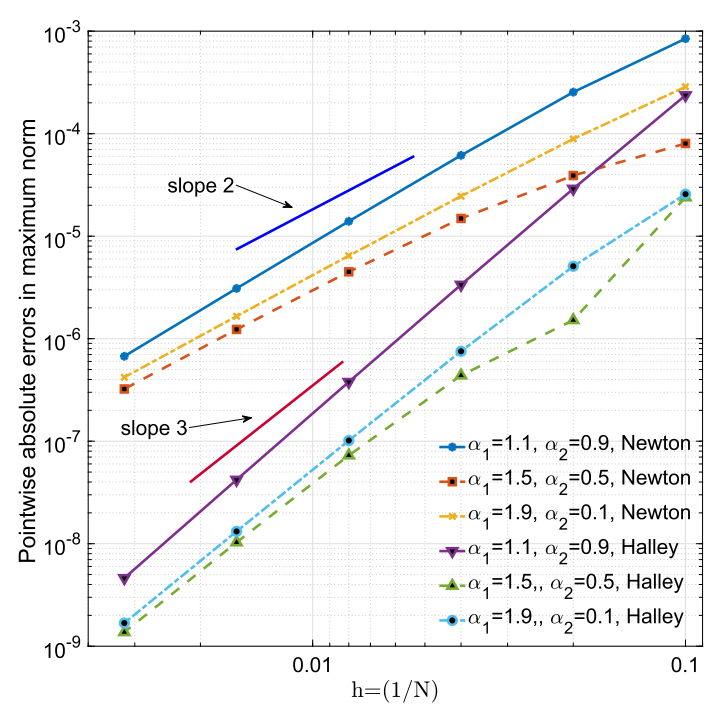

Figure 3 Pointwise absolute errors in maximum norm of Example 4.3 versus the time step $h$ when $\left(\alpha_{1}, \alpha_{2}\right)=(0.9,1.1),(1.5,0.5),(1.9,0.1)$. Graphs demonstrate that computed convergence profiles are nearly $\mathcal{O}\left(h^{2}\right)$ and $\mathcal{O}\left(h^{3}\right)$ for Newton's and Halley's method, respectively

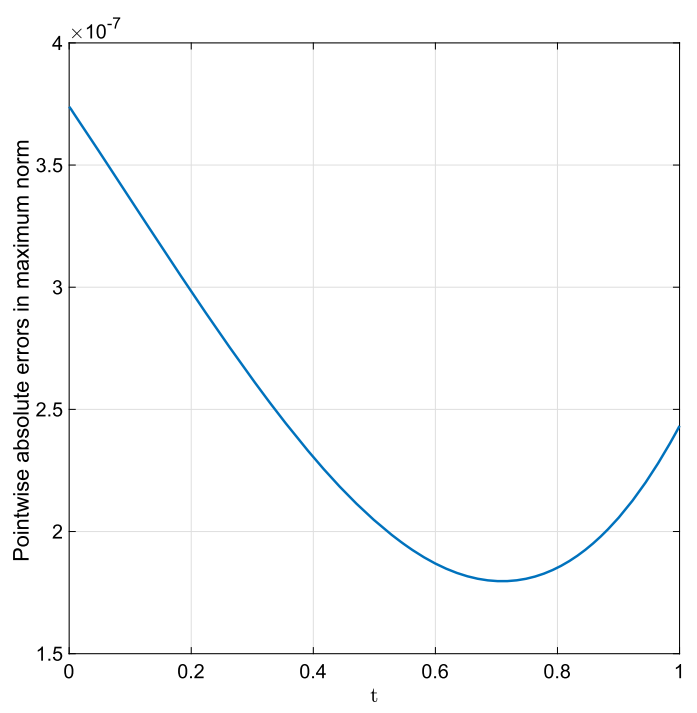

Figure 4 Pointwise absolute errors in maximum norm obtained by the proposed shooting technique with Newton's method of Example 4.3 versus the time step $h$ when $\left(\alpha_{1}, \alpha_{2}\right)=(0.4,1.7), s_{0}=0.2, N=320, m=10$

Figure 6 illustrates convergence profiles obtained by the proposed methods and the modified integral discretization scheme [21] with the variety of fractional orders. From the graphs, we can see that computed rates of convergence are nearly $\mathcal{O}\left(h^{2}\right)$ for the proposed shooting method with Newton's technique and the modified integral discretization scheme [21], $\mathcal{O}\left(h^{3}\right)$ for the proposed shooting method with Halley's scheme, respectively. Plots exhibited in Figs. 7, 8, 9 display pointwise absolute errors in maximum norm versus the time step $h$ and the approximate solution is computed by the modified integral discretization scheme [21] (in Fig. 7), the proposed shooting technique with Newton's 


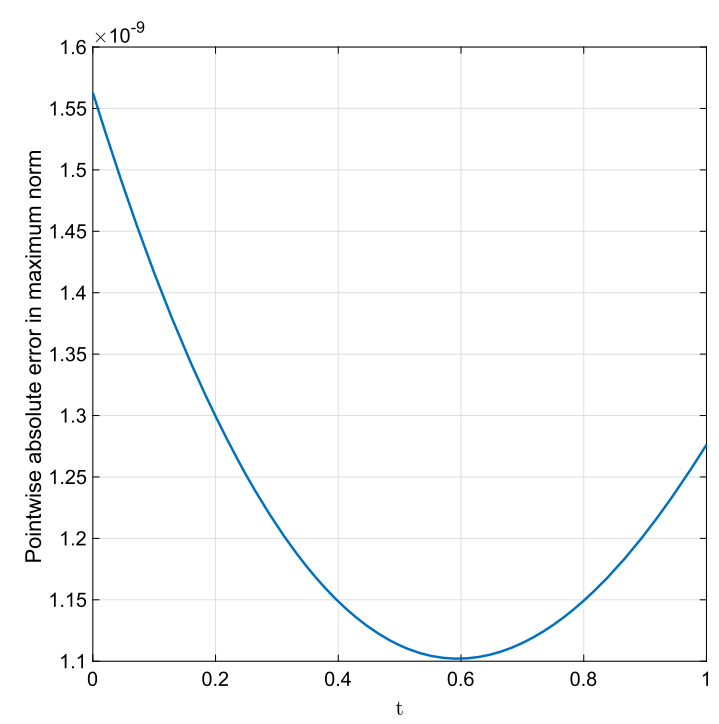

Figure 5 Pointwise absolute errors in maximum norm obtained by the proposed shooting technique with Halley's method of Example 4.3 versus the time step $h$ when $\left(\boldsymbol{\alpha}_{1}, \boldsymbol{\alpha}_{2}\right)=(0.4,1.7), s_{0}=0.2, N=320, m=10$

method (in Fig. 8), the proposed shooting technique with Halley's method (in Fig. 9), respectively.

Example 4.6 Consider the following single-term linear FBVP with RBCs:

$$
\left\{\begin{array}{l}
D_{0}^{\alpha_{2}} y(t)+2 y^{\prime}(t)+3 y(t)=1.25 \\
y(0)-\frac{1}{\alpha_{2}-1} y^{\prime}(0)=0.4, \quad y(1)=1.7
\end{array}\right.
$$

where $1<\alpha_{2}<2$.

The exact solution of Example 4.6 is unknown so we alter the measure to estimate the errors employing the uniform two-mesh differences and orders of convergence introduced in [22]:

$$
e_{N}=\max _{1 \leq j \leq N}\left|y_{j}^{c}-y_{2 j}^{c}\right|
$$

where $y_{2 j}^{c}$ denotes the approximate solution at $t_{2 j}$ by the proposed methods with $h=1 / 2 N$. Using the errors $e_{N}$, we estimate the convergence profiles as follows:

$$
r_{N}=\log _{2}\left(\frac{e_{N}}{e_{2 N}}\right)
$$

In this example, we compare performances of our proposed shooting methods with the finite difference method proposed in [22] and the numerical results are shown in Table 12. We observe the following from the results:

1 In Table 12, uniform two-mesh difference errors and convergence rates computed by (a) the finite difference method [22], 


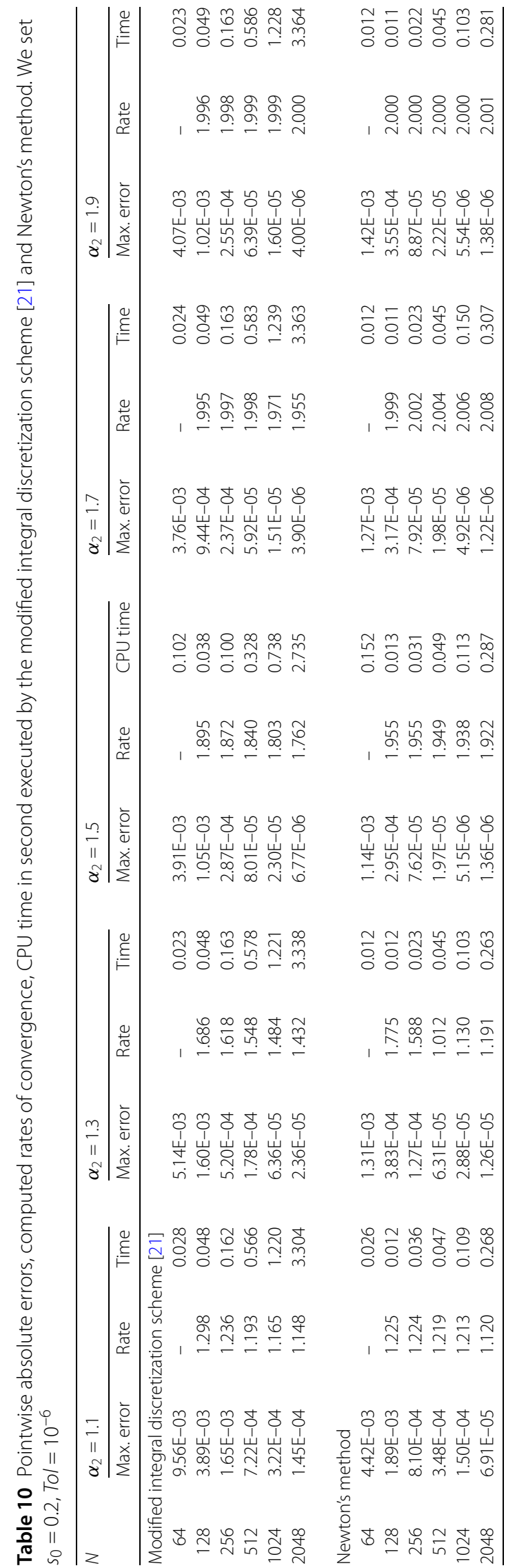




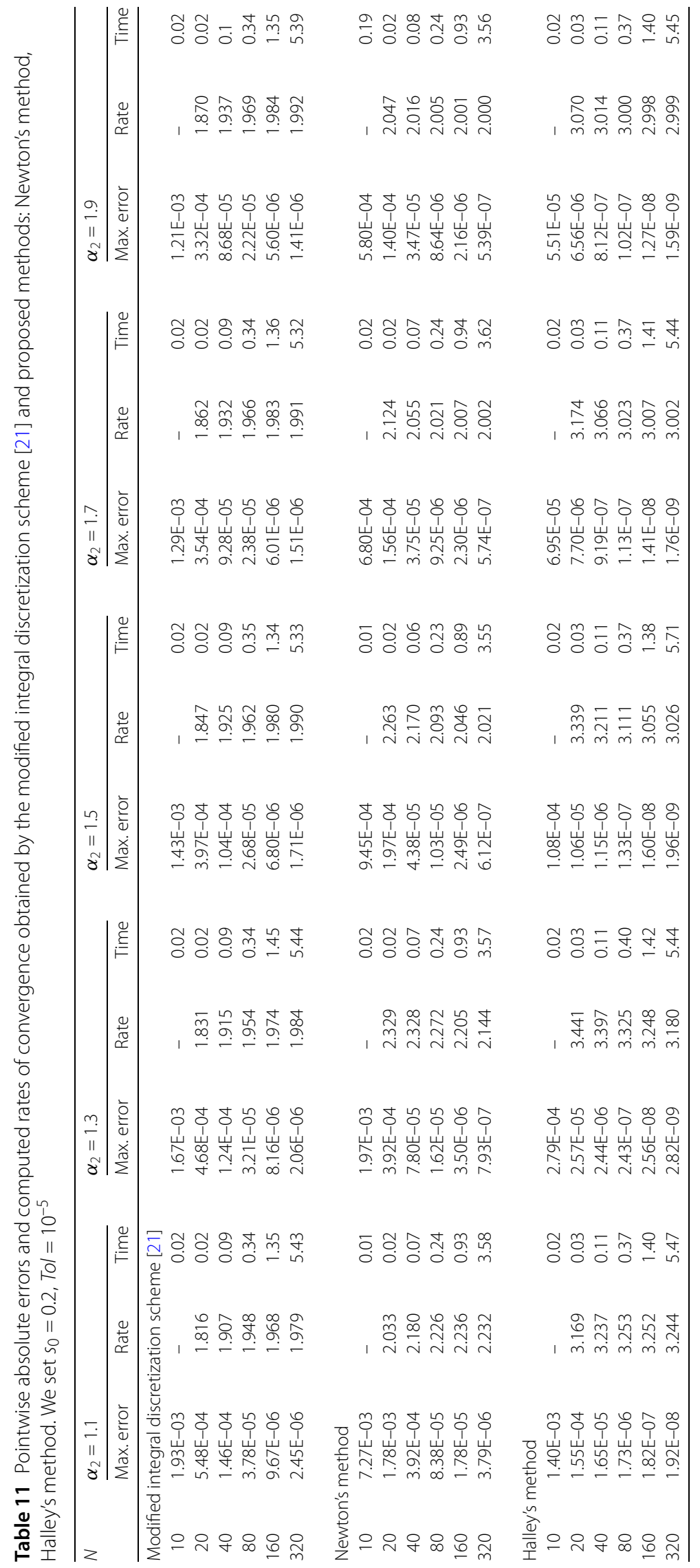




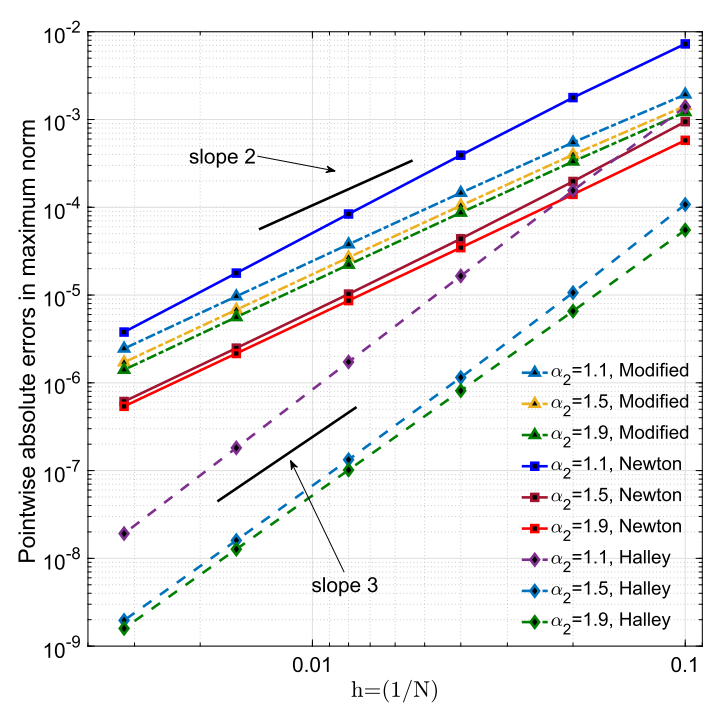

Figure 6 Pointwise absolute errors in maximum norm of Example 4.5 versus the time step $h$ when $\alpha_{2}=1.1,1.5,1.9$. Graphs demonstrate that computed convergence profiles are nearly $\mathcal{O}\left(h^{2}\right)$ for Newton's and modified integral discretization scheme [21], $\mathcal{O}\left(h^{3}\right)$ for Halley's method, respectively

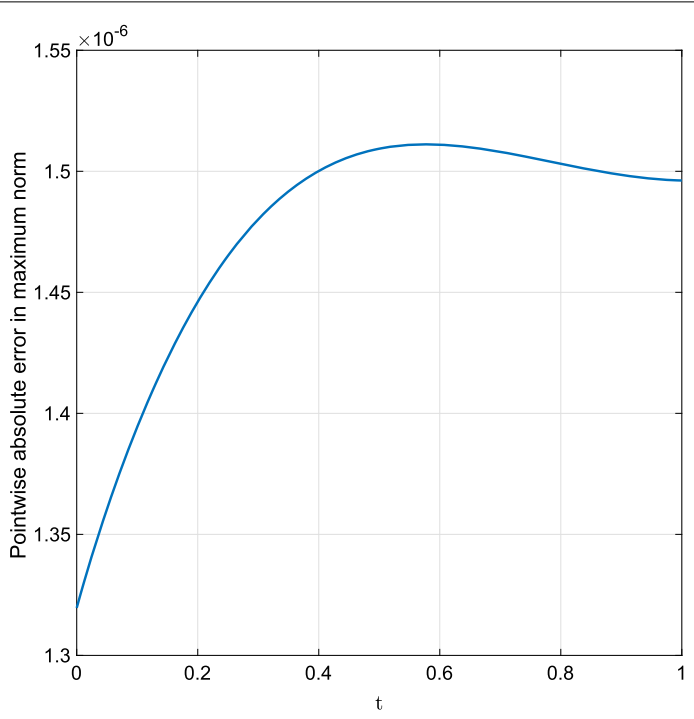

Figure 7 Pointwise absolute errors in maximum norm obtained by the modified integral discretization scheme [21] of Example 4.5 versus the time step $h$ when $\left(\alpha_{1}, \alpha_{2}\right)=\left(1-10^{-16}, 1.7\right), s_{0}=0.2, N=320, T_{0} /=10^{-5}$

(b) the proposed shooting technique based on Newton's method with linear explicit method described in Appendix A,

(c) the proposed shooting technique based on Newton's method with HPCM,

(d) the proposed shooting technique based on Halley's method with HPCM versus the number of time step $N$ as $\alpha_{2}=1.3,1.5,1.7,1.9$ are demonstrated. We set $s_{0}=0.2, T o l=10^{-10}$ as our proposed methods are implemented.

2 It is evident that the uniform two-mesh difference errors computed by our proposed methods are less than the finite difference method [22] for all $N$ and $\alpha_{2}$. 


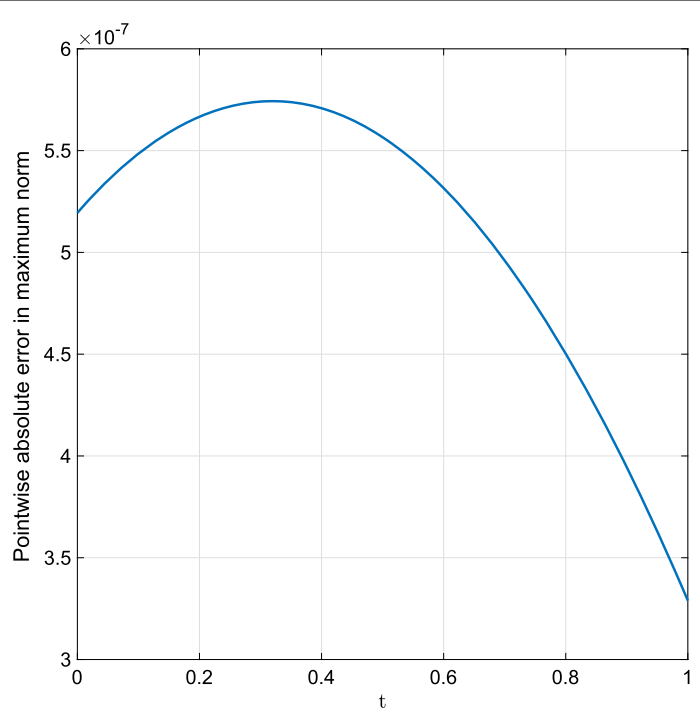

Figure 8 Pointwise absolute errors in maximum norm obtained by the proposed shooting technique with Newton's method of Example 4.5 versus the time step $h$ when $\left(\alpha_{1}, \alpha_{2}\right)=\left(1-10^{-16}, 1.7\right), s_{0}=0.2, N=320$, $\mathrm{TO}=10^{-5}$

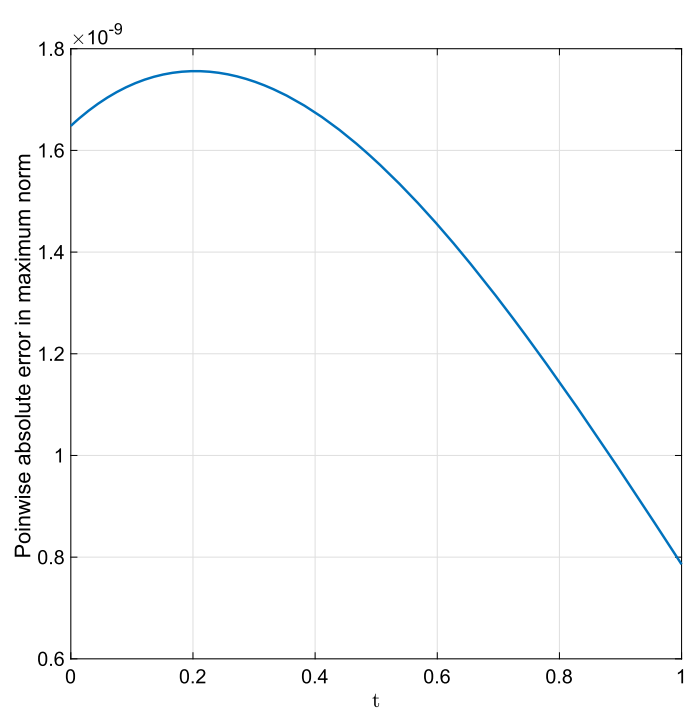

Figure 9 Poinwise absolute errors in maximum norm obtained by the proposed shooting technique with Halley's method of Example 4.5 versus the time step $h$ when $\left(\alpha_{1}, \alpha_{2}\right)=\left(1-10^{-16}, 1.7\right), s_{0}=0.2, N=320$, $\mathrm{Tol}=10^{-5}$

3 The convergence rates obtained by the proposed method with Newton's method are nearly 2 for all $\alpha_{2}$ but the rates of convergence computed by Halley's method are nearly 3 when $\alpha_{2}=1.3$ only. Nevertheless we can see that the convergence profiles obtained by our proposed methods are higher than the finite difference method [22]. Figure 10 shows two-point difference errors in maximum norms versus the number of step $N$. 
Table 12 The uniform two-mesh difference error and computed rates of convergence obtained by the proposed methods, Newton's method, and Halley's method. We set $s_{0}=0.2, \mathrm{Tol}=10^{-10}$

\begin{tabular}{|c|c|c|c|c|c|c|c|}
\hline \multirow{2}{*}{\multicolumn{3}{|c|}{$\begin{array}{l}\alpha_{2} \quad N=64 \\
\text { Finite difference method [22] }\end{array}$}} & \multirow[t]{2}{*}{$N=128$} & \multirow[t]{2}{*}{$N=256$} & \multirow[t]{2}{*}{$N=512$} & \multirow[t]{2}{*}{$N=1024$} & \multirow[t]{2}{*}{$N=2048$} \\
\hline & & & & & & & \\
\hline 1.3 & $\begin{array}{l}\text { error } \\
\text { rate }\end{array}$ & $\begin{array}{l}6.557 \mathrm{E}-002 \\
0.629\end{array}$ & $\begin{array}{l}4.240 E-002 \\
0.757\end{array}$ & $\begin{array}{l}2.509 E-002 \\
0.855\end{array}$ & $\begin{array}{l}1.387 \mathrm{E}-002 \\
0.919\end{array}$ & $\begin{array}{l}7.337 E-003 \\
0.956\end{array}$ & $\begin{array}{l}3.782 \mathrm{E}-003 \\
0.977\end{array}$ \\
\hline 1.5 & $\begin{array}{l}\text { error } \\
\text { rate }\end{array}$ & $\begin{array}{l}2.271 \mathrm{E}-002 \\
0.844\end{array}$ & $\begin{array}{l}1.265 E-002 \\
0.906\end{array}$ & $\begin{array}{l}6.749 E-003 \\
0.944\end{array}$ & $\begin{array}{l}3.509 E-003 \\
0.967\end{array}$ & $\begin{array}{l}1.795 E-003 \\
0.980\end{array}$ & $\begin{array}{l}9.104 E-004 \\
0.988\end{array}$ \\
\hline 1.7 & $\begin{array}{l}\text { error } \\
\text { rate }\end{array}$ & $\begin{array}{l}1.110 E-002 \\
0.895\end{array}$ & $\begin{array}{l}5.968 E-003 \\
0.927\end{array}$ & $\begin{array}{l}3.140 E-003 \\
0.948\end{array}$ & $\begin{array}{l}1.628 \mathrm{E}-003 \\
0.962\end{array}$ & $\begin{array}{l}8.356 \mathrm{E}-004 \\
0.971\end{array}$ & $\begin{array}{l}4.262 E-004 \\
0.978\end{array}$ \\
\hline 1.9 & $\begin{array}{l}\text { error } \\
\text { rate }\end{array}$ & $\begin{array}{l}5.650 E-003 \\
0.017\end{array}$ & $\begin{array}{l}2.978 \mathrm{E}-003 \\
0.050\end{array}$ & $\begin{array}{l}1.557 E-003 \\
0.084\end{array}$ & $\begin{array}{l}8.090 \mathrm{E}-004 \\
0.117\end{array}$ & $\begin{array}{l}4.184 \mathrm{E}-004 \\
0.168\end{array}$ & $\begin{array}{l}2.156 \mathrm{E}-004 \\
0.255\end{array}$ \\
\hline \multicolumn{8}{|c|}{ Newton's method with linear explicit } \\
\hline 1.3 & $\begin{array}{l}\text { error } \\
\text { rate }\end{array}$ & $\begin{array}{l}5.35 E-03 \\
0.935\end{array}$ & $\begin{array}{l}2.55 \mathrm{E}-03 \\
1.068\end{array}$ & $\begin{array}{l}1.14 \mathrm{E}-03 \\
1.157\end{array}$ & $\begin{array}{l}4.95 \mathrm{E}-04 \\
1.211\end{array}$ & $\begin{array}{l}2.09 \mathrm{E}-04 \\
1.244\end{array}$ & $\begin{array}{l}8.68 \mathrm{E}-05 \\
1.265\end{array}$ \\
\hline 1.5 & $\begin{array}{l}\text { error } \\
\text { rate }\end{array}$ & $\begin{array}{l}5.35 E-03 \\
0.935\end{array}$ & $\begin{array}{l}2.55 \mathrm{E}-03 \\
1.068\end{array}$ & $\begin{array}{l}1.14 \mathrm{E}-03 \\
1.157\end{array}$ & $\begin{array}{l}4.95 \mathrm{E}-04 \\
1.211\end{array}$ & $\begin{array}{l}2.09 \mathrm{E}-04 \\
1.244\end{array}$ & $\begin{array}{l}8.68 \mathrm{E}-05 \\
1.265\end{array}$ \\
\hline 1.7 & $\begin{array}{l}\text { error } \\
\text { rate }\end{array}$ & $\begin{array}{l}4.19 \mathrm{E}-04 \\
1.562\end{array}$ & $\begin{array}{l}1.39 \mathrm{E}-04 \\
1.594\end{array}$ & $\begin{array}{l}4.51 \mathrm{E}-05 \\
1.618\end{array}$ & $\begin{array}{l}1.45 \mathrm{E}-05 \\
1.636\end{array}$ & $\begin{array}{l}4.62 \mathrm{E}-06 \\
1.650\end{array}$ & $\begin{array}{l}1.46 \mathrm{E}-06 \\
1.660\end{array}$ \\
\hline 1.9 & $\begin{array}{l}\text { error } \\
\text { rate }\end{array}$ & $\begin{array}{l}1.00 \mathrm{E}-04 \\
1.798\end{array}$ & $\begin{array}{l}2.85 \mathrm{E}-05 \\
1.810\end{array}$ & $\begin{array}{l}\text { 8.07E-06 } \\
1.821\end{array}$ & $\begin{array}{l}2.27 \mathrm{E}-06 \\
1.830\end{array}$ & $\begin{array}{l}6.35 \mathrm{E}-07 \\
1.837\end{array}$ & $\begin{array}{l}1.77 \mathrm{E}-07 \\
1.844\end{array}$ \\
\hline \multicolumn{8}{|c|}{ Newton's method } \\
\hline 1.3 & $\begin{array}{l}\text { error } \\
\text { rate }\end{array}$ & $\begin{array}{l}4.04 \mathrm{E}-03 \\
2.238\end{array}$ & $\begin{array}{l}6.03 E-04 \\
2.745\end{array}$ & $\begin{array}{l}4.95 E-05 \\
3.607\end{array}$ & $\begin{array}{l}8.12 \mathrm{E}-06 \\
2.606\end{array}$ & $\begin{array}{l}5.88 \mathrm{E}-06 \\
0.465\end{array}$ & $\begin{array}{l}2.17 E-06 \\
1.438\end{array}$ \\
\hline 1.5 & $\begin{array}{l}\text { error } \\
\text { rate }\end{array}$ & $\begin{array}{l}2.41 \mathrm{E}-04 \\
0.329\end{array}$ & $\begin{array}{l}8.57 \mathrm{E}-05 \\
1.489\end{array}$ & $\begin{array}{l}2.50 \mathrm{E}-05 \\
1.775\end{array}$ & $\begin{array}{l}6.74 \mathrm{E}-06 \\
1.893\end{array}$ & $\begin{array}{l}1.74 \mathrm{E}-06 \\
1.949\end{array}$ & $\begin{array}{l}4.42 \mathrm{E}-07 \\
1.980\end{array}$ \\
\hline 1.7 & $\begin{array}{l}\text { error } \\
\text { rate }\end{array}$ & $\begin{array}{l}1.53 \mathrm{E}-04 \\
1.779\end{array}$ & $\begin{array}{l}4.05 \mathrm{E}-05 \\
1.919\end{array}$ & $\begin{array}{l}1.03 \mathrm{E}-05 \\
1.975\end{array}$ & $\begin{array}{l}2.58 \mathrm{E}-06 \\
1.998\end{array}$ & $\begin{array}{l}6.40 \mathrm{E}-07 \\
2.010\end{array}$ & $\begin{array}{l}1.58 \mathrm{E}-07 \\
2.017\end{array}$ \\
\hline 1.9 & $\begin{array}{l}\text { error } \\
\text { rate }\end{array}$ & $\begin{array}{l}9.08 \mathrm{E}-05 \\
1.941\end{array}$ & $\begin{array}{l}2.29 \mathrm{E}-05 \\
1.984\end{array}$ & $\begin{array}{l}5.74 \mathrm{E}-06 \\
1.997\end{array}$ & $\begin{array}{l}1.43 \mathrm{E}-06 \\
2.001\end{array}$ & $\begin{array}{l}3.58 \mathrm{E}-07 \\
2.003\end{array}$ & $\begin{array}{l}8.92 E-08 \\
2.004\end{array}$ \\
\hline \multicolumn{8}{|c|}{ Halley's method } \\
\hline 1.3 & $\begin{array}{l}\text { error } \\
\text { rate }\end{array}$ & $\begin{array}{l}1.00 E-03 \\
3.056\end{array}$ & $\begin{array}{l}9.19 \mathrm{E}-05 \\
3.456\end{array}$ & $\begin{array}{l}6.67 E-06 \\
3.782\end{array}$ & $\begin{array}{l}3.25 E-07 \\
4.358\end{array}$ & $\begin{array}{l}1.60 \mathrm{E}-08 \\
4.348\end{array}$ & $\begin{array}{l}8.44 \mathrm{E}-09 \\
0.920\end{array}$ \\
\hline 1.5 & $\begin{array}{l}\text { error } \\
\text { rate }\end{array}$ & $\begin{array}{l}8.24 \mathrm{E}-06 \\
2.111\end{array}$ & $\begin{array}{l}2.48 \mathrm{E}-06 \\
1.732\end{array}$ & $\begin{array}{l}8.70 \mathrm{E}-07 \\
1.511\end{array}$ & $\begin{array}{l}3.02 E-07 \\
1.524\end{array}$ & $\begin{array}{l}1.05 E-07 \\
1.526\end{array}$ & $\begin{array}{l}3.64 \mathrm{E}-08 \\
1.525\end{array}$ \\
\hline 1.7 & $\begin{array}{l}\text { error } \\
\text { rate }\end{array}$ & $\begin{array}{l}1.26 \mathrm{E}-05 \\
1.862\end{array}$ & $\begin{array}{l}3.60 \mathrm{E}-06 \\
1.802\end{array}$ & $\begin{array}{l}1.07 E-06 \\
1.746\end{array}$ & $\begin{array}{l}3.26 \mathrm{E}-07 \\
1.719\end{array}$ & $\begin{array}{l}9.97 \mathrm{E}-08 \\
1.707\end{array}$ & $\begin{array}{l}3.06 \mathrm{E}-08 \\
1.703\end{array}$ \\
\hline 1.9 & $\begin{array}{l}\text { error } \\
\text { rate }\end{array}$ & $\begin{array}{l}4.87 E-06 \\
2.365\end{array}$ & $\begin{array}{l}1.06 \mathrm{E}-06 \\
2.200\end{array}$ & $\begin{array}{l}2.53 E-07 \\
2.063\end{array}$ & $\begin{array}{l}6.42 \mathrm{E}-08 \\
1.980\end{array}$ & $\begin{array}{l}1.68 \mathrm{E}-08 \\
1.937\end{array}$ & $\begin{array}{l}\text { 4.44E-09 } \\
1.916\end{array}$ \\
\hline
\end{tabular}

\section{Conclusion}

We introduced new numerical schemes for solving FNBVPs with any RBCs. The idea was to transform a FNBVP into a system of FIVPs. By doing that we could adopt a pre-existing numerical method for solving the system of FIVPs and we mainly employed HPCMs. The unknown IC $s$ in the system was approximated by the proposed shooting methods based on Newton's and Halley's method and this is the main algorithm of the proposed schemes. Under the assumption that $m$ is large enough so that $\left|\tilde{F}\left(s_{m}\right)\right|$ is small enough, theoretical convergence rates of proposed methods were $\mathcal{O}\left(h^{2}\right)$ for shooting with Newton's method and $\mathcal{O}\left(h^{3}\right)$ for shooting with Halley's method on account of global error estimates in HPCMs.

In Examples 4.1 through 4.3, we verified that the proposed schemes can handle doubleterm FNVBPs with RBCs whose exact solutions include polynomial, exponential, and sine 


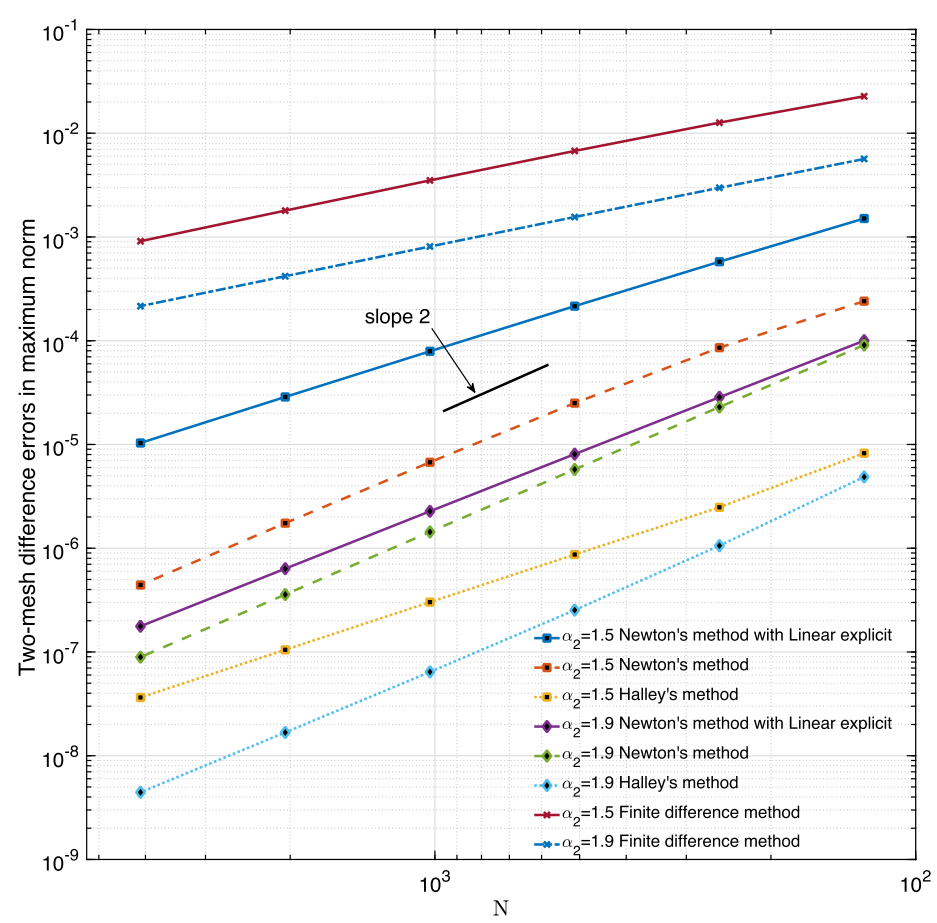

Figure 10 Two-mesh difference errors in maximum norm obtained by the proposed methods and a finite difference [22] of Example 4.6 versus the number of step $N$ when $\left(\alpha_{1}, \alpha_{2}\right)=\left(1-10^{-16}, 1.5\right),\left(1-10^{-16}, 1.9\right)$ $s_{0}=0.2, T O l=10^{-5}$

function. Convergence profiles obtained by the proposed schemes were computed as expected by the global error estimates. However, Tables 3, 6, and 9 suggest that the convergence rate of the sequence $\left\{s_{k}\right\}$ depends on fractional orders. We still need to address an error analysis of shooting techniques based on Newton's and Halley's methods for solving a system of FIVPs. This will be considered in a subsequent paper. Examples 4.4 and 4.5 demonstrated the performance of proposed methods for solving single-term linear FBVPs with exact solutions having low regularity and high regularity, respectively. Tables 10 and 11 showed that the proposed methods can deal with not only nonlinear FBVPs but also linear FBVPs. In Example 4.4, we adopted the linear explicit method described in Appendix $A$ and this shows that the proposed shooting techniques can be assembled with not only HPCMs but also other pre-existing numerical schemes for solving a system of FIVPs. In Example 4.5, we observed that computed convergence rates obtained by our proposed shooting technique based on Halley's method with third-order HPCM are higher than the modified integral discretization scheme [21].

\section{Appendix A: Linear explicit method}

Let us consider the following linear single-term FBVP with RBCs:

$$
\left\{\begin{array}{l}
D_{0}^{\alpha_{2}} y(t)=f(t)+c(t) y(t)+b(t) y^{\prime}(t), \\
a_{1} y(0)+b_{1} y^{\prime}(0)=\gamma_{1}, \quad a_{2} y(1)+b_{2} y^{\prime}(1)=\gamma_{2},
\end{array}\right.
$$


where $1<\alpha_{2}<2$. By Lemmas 2.1 through 2.4 and Theorem 2.1, the FBVP (36) is equivalent to the following system:

$$
\begin{cases}D_{0}^{1-\epsilon} y(t)=z(t) & y(0)=s \\ D_{0}^{\alpha} z(t)=f(t)+c(t) y(t)+b(t) z(t) & z(0)=\left(\gamma_{1}-a_{1} s\right) / b_{1} .\end{cases}
$$

Expressing the solution of (37) as the discretized form of the Volterra integral equation, which is equivalent to (37), we obtain

$$
\begin{aligned}
y\left(t_{n+1}\right)= & y(0)+\frac{1}{\Gamma(1-\epsilon)} \int_{t_{0}}^{t_{n+1}}\left(t_{n+1}-\tau\right)^{-\epsilon} z(\tau) d \tau, \\
z\left(t_{n+1}\right)= & z(0)+J_{0}^{\alpha_{2}-1} f\left(t_{n+1}\right) \\
& +\frac{1}{\Gamma\left(\alpha_{2}\right)} \int_{t_{0}}^{t_{n+1}}\left(t_{n+1}-\tau\right)^{\alpha_{2}-1}(c(\tau) y(\tau)+b(\tau) z(\tau)) d \tau .
\end{aligned}
$$

The approximation solutions to $y\left(t_{n+1}\right)$ and $z\left(t_{n+1}\right)$ in (38) with $s_{k}$ can be explicitly described as follows:

$$
\begin{aligned}
y_{n+1}= & s_{k}+\frac{1}{\Gamma(1-\epsilon)} \sum_{j=0}^{n} \int_{t_{j}}^{t_{j+1}}\left(t_{n+1}-\tau\right)^{-\epsilon} z(\tau) d \tau, \\
z_{n+1}= & \frac{\gamma_{1}-a_{1} s_{k}}{b_{1}}+J_{0}^{\alpha_{2}-1} f\left(t_{n+1}\right) \\
& +\frac{1}{\Gamma\left(\alpha_{2}\right)} \sum_{j=0}^{n} \int_{t_{j}}^{t_{j+1}}\left(t_{n+1}-\tau\right)^{\alpha_{2}-1}(c(\tau) y(\tau)+b(\tau) z(\tau)) d \tau .
\end{aligned}
$$

Replacing $y(\tau)$ and $z(\tau)$ with linear interpolation, we have

$$
\begin{aligned}
y_{n+1}= & s_{k}+\frac{1}{\Gamma(1-\epsilon)} \sum_{j=0}^{n} \int_{t_{j}}^{t_{j+1}}\left(t_{n+1}-\tau\right)^{-\epsilon}\left(\frac{t_{j+1}-\tau}{h} z_{j}+\frac{t_{j}-\tau}{-h} z_{j+1}\right) d \tau, \\
z_{n+1}= & \frac{\gamma_{1}-a_{1} s_{k}}{b_{1}}+J_{0}^{\alpha_{2}-1} f\left(t_{n+1}\right) \\
& +\frac{1}{\Gamma\left(\alpha_{2}\right)} \sum_{j=0}^{n} \int_{t_{j}}^{t_{j+1}}\left(t_{n+1}-\tau\right)^{\alpha_{2}-1}\left\{\frac{t_{j+1}-\tau}{h}\left(c\left(t_{j}\right) y_{j}+b\left(t_{j}\right) z_{j}\right)\right. \\
& \left.+\frac{t_{j}-\tau}{-h}\left(c\left(t_{j+1}\right) y_{j+1}+b\left(t_{j+1}\right) z_{j+1}\right)\right\} d \tau .
\end{aligned}
$$

Let us shorten the expression of $y_{n+1}, z_{n+1}$ in (39) as follows:

$$
y_{n+1}=s_{k}+\sum_{j=0}^{n}\left[A_{j}^{1} z_{j}+A_{j}^{2} z_{j+1}\right]
$$




$$
\begin{aligned}
z_{n+1}= & \frac{\gamma_{1}-a_{1} s_{k}}{b_{1}}+J_{0}^{\alpha_{2}-1} f\left(t_{n+1}\right) \\
& +\sum_{j=0}^{n-1}\left[B_{j}^{1}\left(c\left(t_{j}\right) y_{j}+b\left(t_{j}\right) z_{j}\right)+B_{j}^{2}\left(c\left(t_{j+1}\right) y_{j+1}+b\left(t_{j+1}\right) z_{j+1}\right)\right] \\
& +B_{n}^{1}\left(c\left(t_{n}\right) y_{n}+b\left(t_{n}\right) z_{n}\right)+B_{n}^{2}\left(c\left(t_{n+1}\right) y_{n+1}+b\left(t_{n+1}\right) z_{n+1}\right) .
\end{aligned}
$$

We omit describing the definitions of $A_{j}^{i}, B_{j}^{i}, i=1,2, j=0, \ldots, n$, because that is straightforward. Substituting the explicit form of $y_{n+1}$ into the right-hand side of $z_{n+1}$, we have

$$
\begin{aligned}
z_{n+1}= & \frac{\gamma_{1}-a_{1} s_{k}}{b_{1}}+J_{0}^{\alpha_{2}-1} f\left(t_{n+1}\right) \\
& +\sum_{j=0}^{n-1}\left[B_{j}^{1}\left(c\left(t_{j}\right) y_{j}+b\left(t_{j}\right) z_{j}\right)+B_{j}^{2}\left(c\left(t_{j+1}\right) y_{j+1}+b\left(t_{j+1}\right) z_{j+1}\right)\right] \\
& +B_{n}^{1}\left(c\left(t_{n}\right) y_{n}+b\left(t_{n}\right) z_{n}\right) \\
& +B_{n}^{2}\left[c\left(t_{n+1}\right)\left\{s_{k}+\sum_{j=0}^{n}\left(A_{j}^{1} z_{j}+A_{j}^{2} z_{j+1}\right)\right\}+b\left(t_{n+1}\right) z_{n+1}\right] \\
= & \frac{\gamma_{1}-a_{1} s_{k}}{b_{1}}+J_{0}^{\alpha_{2}-1} f\left(t_{n+1}\right) \\
& +\sum_{j=0}^{n-1}\left[B_{j}^{1}\left(c\left(t_{j}\right) y_{j}+b\left(t_{j}\right) z_{j}\right)+B_{j}^{2}\left(c\left(t_{j+1}\right) y_{j+1}+b\left(t_{j+1}\right) z_{j+1}\right)\right] \\
& +B_{n}^{1}\left(c\left(t_{n}\right) y_{n}+b\left(t_{n}\right) z_{n}\right) \\
& +B_{n}^{2} c\left(t_{n+1}\right)\left\{s_{k}+\sum_{j=0}^{n-1}\left(A_{j}^{1} z_{j}+A_{j}^{2} z_{j+1}\right)+A_{n}^{1} z_{n}\right\} \\
& +\left(B_{n}^{2} A_{n}^{2} c\left(t_{n+1}\right)+b\left(t_{n+1}\right)\right) z_{n+1} .
\end{aligned}
$$

Since the right-hand side of (40) is linear in $z_{j}, j=0, \ldots, n+1, z_{n+1}$ can be explicitly expressed as follows:

$$
\begin{aligned}
z_{n+1}= & {\left[\frac{\gamma_{1}-a_{1} s_{k}}{b_{1}}+J_{0}^{\alpha_{2}-1} f\left(t_{n+1}\right)+\sum_{j=0}^{n-1}\left[B_{j}^{1}\left(c\left(t_{j}\right) y_{j}+b\left(t_{j}\right) z_{j}\right)\right.\right.} \\
& \left.+B_{j}^{2}\left(c\left(t_{j+1}\right) y_{j+1}+b\left(t_{j+1}\right) z_{j+1}\right)\right]+B_{n}^{1}\left(c\left(t_{n}\right) y_{n}+b\left(t_{n}\right) z_{n}\right) \\
& \left.+B_{n}^{2} c\left(t_{n+1}\right)\left\{s_{k}+\sum_{j=0}^{n-1}\left(A_{j}^{1} z_{j}+A_{j}^{2} z_{j+1}\right)+A_{n}^{1} z_{n}\right\}\right] \\
& /\left[1-\left\{B_{n}^{2} A_{n}^{2} c\left(t_{n+1}\right)+b\left(t_{n+1}\right)\right\}\right] .
\end{aligned}
$$

\section{Appendix B: Step of algorithm}

Steps of the proposed algorithms for solving the FNVBP (7)

Input parameters: fractional orders $\alpha_{1}, \alpha_{2}$; endpoints $0, b$; RBCs $a_{1}, b_{1}, \gamma_{1}, a_{2}, b_{2}, \gamma_{2}$; number of time sub-intervals $N$; maximum number of iterations in Newton's and Halley's methods $m$; initial approximation of $s s_{0}$; tolerance $T o l$. 
Step 1) Set $h=b / N, t_{n}=n \cdot h, k=0$.

Step 2) While $(k \leq m)$ do Steps $3 \cdots 6$.

Step 3) For $n=0, \ldots, N-1$ do Steps $3-1 \cdots 3-3$.

3 - 1) Compute predictors $y_{n+1}^{p}, w_{n+1}^{p}, z_{n+1}^{p}$ using the formulas (33) (Newton's method) or (35) (Halley's method) with $s_{k}$ individually.

3 - 2) Compute $f_{n+1}^{p} \equiv f\left(t_{n+1}, y_{n+1}^{p}, w_{n+1}^{p}\right)$.

3 -3) Compute correctors $y_{n+1}^{c}, w_{n+1}^{c}, z_{n+1}^{c}$ using the formulas (32) (Newton's method) or (34) (Halley's method) individually.

Step 4) Compute the approximated error function with $s_{k}$ :

$$
\left|\tilde{F}\left(s_{k}\right)\right|=\left|a_{2} y_{N}^{c}\left(s_{k}\right)+b_{2} z_{N}^{c}\left(s_{k}\right)-\gamma_{2}\right| .
$$

If $\left|\tilde{F}\left(s_{k}\right)\right| \leq$ Tol then STOP.

Do Step 5 to compute $F_{s}\left(s_{k}\right)$ (Newton's or Halley's method) and $F_{s s}\left(s_{k}\right)$ (Halley's method).

Step 5) For $n=0, \ldots, N-1$ do Steps $5-1 \cdots 5-5$.

5 - 1) Compute predictors $y_{s, n+1}^{p}, w_{s, n+1}^{p}, z_{s, n+1}^{p}$ in (25) using the formulas (33) (Newton's method) or (35) (Halley's method) individually.

5 - 2) Compute predictors $y_{s s, n+1}^{p}, w_{s s, n+1}^{p}, z_{s s, n+1}^{p}$ in (28) using the formulas (35) (Halley's method) individually. (Skip step for Newton's method.)

5 - 3) Compute $f_{s, n+1}^{p}$ defined by (26) (Newton's and Halley's methods). Compute $f_{s s, n+1}^{p}$ defined by (29) (Halley's method).

5 -4) Compute correctors $y_{s, n+1}^{c}, w_{s, n+1}^{c}, z_{s, n+1}^{c}$ in (25) using the formulas (32) (Newton's method) or (35) (Halley's method) individually.

5 - 5) Compute predictors $y_{s s, n+1}^{c}, w_{s s, n+1}^{c}, z_{s s, n+1}^{c}$ in (28) using the formulas (34) (Halley's method) individually. (Skip step for Newton's method.)

Step 6) Compute $s_{k+1}$ using the formulas (23) (Newton's method) or (27) (Halley's method).

$$
k=k+1 \text {. }
$$

\section{Acknowledgements}

We would like to thank the editors of the journal as well as the anonymous reviewers for their valuable suggestions that make the manuscript more consistent.

\section{Funding}

This work was supported by the National Research Foundation of Korea(NRF) grant funded by the Korea government(MSIP) (NRF-2016R1D1A1B03935514, NRF-2019R1A2C1005142)

\section{Availability of data and materials}

Not applicable.

\section{Competing interests}

The authors declare that they have no competing interests. 
Authors' contributions

HK: Methodology, Writing — Original and Revision draft, JL: Data curation, Simulation, BJ: Conceptualization of this study, Analysis, Writing - Original and Revision draft. All authors read and approved the final manuscript.

\section{Author details}

'Department of Mathematics, North Greenville University, 29688 Tigerville, SC, USA. ${ }^{2}$ Department of Mathematical

Sciences, Ulsan National Institute of Science and Technology (UNIST), 44919, Ulsan, Republic of Korea.

\section{Publisher's Note}

Springer Nature remains neutral with regard to jurisdictional claims in published maps and institutional affiliations.

\section{Received: 29 October 2020 Accepted: 24 March 2021 Published online: 01 April 2021}

\section{References}

1. Nguyen, T.B., Jang, B.: A high-order predictor-corrector method for solving nonlinear differential equations of fractional order. Fract. Calc. Appl. Anal. 20(2), 447-476 (2017)

2. Caputo, M.: Linear model of dissipation whose $\mathrm{Q}$ is almost frequency independent-II. Geophys. J. R. Astron. Soc. 13, 529-539 (1967)

3. Dalir, M., Bashour, M.: Applications of fractional calculus. Appl. Math. Sci. 4, 1021-1032 (2010)

4. Das, S.: Functional Fractional Calculus for System Identification and Controls. Springer, Berlin (2008)

5. Hilfer, R.: Applications of Fractional Calculus in Physics. World Scientific, Singapore (2000)

6. Magin, R.L.: Fractional Calculus in Bioengineering. Begell House Publishers (2006)

7. Podlubny, I.: Fractional Differential Equations. Academic Press, San Diego (1999)

8. Arqub, O.A., Shaqagfeh, N.: Application of reproducing kernel algorithm for solving Dirichlet time-fractional diffusion-Gordon types equations in porous media. J. Porous Media 22(4), 411-434 (2019)

9. Arqub, O.A.: Numerical algorithm for the solutions of fractional order systems of Dirichlet function types with comparative analysis. Fundam. Inform. 166(2), 111-137 (2019)

10. Arqub, O.A.: Application of residual power series method for the solution of time-fractional Schrödinger equations in one-dimensional space. Fundam. Inform. 166(2), 87-110 (2019)

11. Roul, P., Goura, V.M.K.P.: A high order numerical scheme for solving a class of non-homogeneous time-fractional reaction diffusion equation. Numer. Methods Partial Differ. Equ. https://doi.org/10.1002/num.22594

12. Roul, P., Rohil, V., Espinosa-Paredes, G., Goura, V.M.K.P., Gedam, R.S., Obaidurrahman, K.: Design and analysis of a numerical method for fractional neutron diffusion equation with delayed neutrons. Appl. Numer. Math. 157, 634-653 (2020)

13. Roul, P., Goura, V.M.K.P.: A high-order b-spline collocation scheme for solving a nonhomogeneous time-fractional diffusion equation. Math. Methods Appl. Sci. 44(1), 546-567 (2020)

14. Roul, P., Rohil, V., Espinosa-Paredes, G., Obaidurrahman, K.: An efficient numerical method for fractional neutron diffusion equation in the presence of different types of reactivities. Ann. Nucl. Energy 152, 108038 (2021)

15. Baleanu, D., Etemad, S., Rezapour, S.: A hybrid Caputo fractional modeling for thermostat with hybrid boundary value conditions. Bound. Value Probl. 2020(1), 64 (2020). https://doi.org/10.1186/s13661-020-01361-0

16. Patnaik, S., Sidhardh, S., Semperlotti, F.: A Ritz-based finite element method for a fractional-order boundary value problem of nonlocal elasticity. Int. J. Solids Struct. 202, 398-417 (2020)

17. Cai, H., An, Q.: A fractional spectral collocation method for general Caputo two-point boundary value problems. Appl. Numer. Math. (2021). https://doi.org/10.1016/j.apnum.2021.01.006

18. Liu, L.-B., Liang, Z., Long, G., Liang, Y.: Convergence analysis of a finite difference scheme for a Riemann-Liouville fractional derivative two-point boundary value problem on an adaptive grid. J. Comput. Appl. Math. 375, 112809 (2020). https://doi.org/10.1016/j.cam.2020.112809

19. Diethelm, K.: The Analysis of Fractional Differential Equations: An Application-Oriented Exposition Using Differential Operators of Caputo Type. Springer, Berlin (2010)

20. Cen, Z., Huang, J., Le, A.: A modified integral discretization scheme for a two-point boundary value problem with a Caputo fractional derivative. J. Comput. Appl. Math. 367(15), 112465 (2020)

21. Cen, Z., Huang, J., Xu, A.: An efficient numerical method for a two-point boundary value problem with a Caputo fractional derivative an efficient numerical method for a two-point boundary value problem with a Caputo fractional derivative. J. Comput. Appl. Math. 336, 1-7 (2018)

22. Stynes, M., Gracia, J.L.: A finite difference method for a two-point boundary value problem with a Caputo fractional derivative. IMA J. Numer. Anal. 35, 698-721 (2015) 